\title{
Documenting a Rare Tornado Merger Observed in the 24 May 2011 EI Reno-Piedmont, Oklahoma, Supercell*
}

\author{
Michael M. French, ${ }^{+}$PATRick S. Skinner, And Louis J. Wicker \\ NOAA/National Severe Storms Laboratory, Norman, Oklahoma \\ HOWARD B. BLUESTEIN \\ School of Meteorology, University of Oklahoma, Norman, Oklahoma
}

(Manuscript received 21 October 2014, in final form 21 April 2015)

\begin{abstract}
Unique observations of the interaction and likely merger of two cyclonic tornadoes are documented. One of the tornadoes involved in the interaction was the enhanced Fujita scale (EF5) El Reno-Piedmont, Oklahoma, tornado from 24 May 2011 and the other was a previously undocumented tornado. Data from three S-band radars: Twin Lakes, Oklahoma (KTLX); Norman, Oklahoma (KOUN); and the multifunction phased-array radar (MPAR), are used to detail the formation of the second tornado, which occurred to the northwest of the original tornado in an area of strong radial convergence. Radar data and isosurfaces of azimuthal shear provide evidence that both tornadoes formed within an elongated area of mesocyclone-scale cyclonic rotation. The path taken by the primary tornado and the formation location of the second tornado are different from previous observations of simultaneous cyclonic tornadoes, which have been most often observed in the cyclic tornadogenesis process. The merger of the two tornadoes occurred during the sampling period of a mobile phased-array radar-the Mobile Weather Radar, 2005 X-Band, Phased Array (MWR-05XP). MWR-05XP electronic scanning in elevation allowed for the merger process to be examined up to $4 \mathrm{~km}$ above radar level every $11 \mathrm{~s}$. The tornadic vortex signatures (TVSs) associated with the tornadoes traveled around each other in a counterclockwise direction then merged in a helical manner up through storm midlevels. Upon merging, both the estimated intensity and size of the TVS associated with the resulting tornado increased dramatically. Similarities between the merger observed in this case and in previous cases also are discussed.
\end{abstract}

\section{Introduction}

On 24 May 2011, a series of supercells produced at least 12 tornadoes in central Oklahoma, including an enhanced Fujita scale (EF5) tornado having a pathlength of $101 \mathrm{~km}$ near El Reno and Piedmont, Oklahoma, among other locations, over a 105-min span [hereafter the El

\footnotetext{
* Supplemental information related to this paper is available at the Journals Online website: http://dx.doi.org/10.1175/MWR-D-1400349.s1.

${ }^{+}$Current affiliation: School of Marine and Atmospheric Sciences, Stony Brook University, Stony Brook, New York.

Corresponding author address: Michael M. French, School of Marine and Atmospheric Sciences, Stony Brook University, Stony Brook, NY 11794

E-mail: michael.m.french@stonybrook.edu
}

Reno-Piedmont tornado (ERPT); Pazmany et al. (2013)]. At least two times during the ERPT life cycle, visual observations and radar data support the existence of a second cyclonic tornado in close proximity to the ERPT (Storm Data; French et al. 2014). There are several examples of simultaneous cyclonic supercell tornadoes documented in the literature, many of which are those observed during the cyclic tornadogenesis process (e.g., Burgess et al. 1982; Dowell and Bluestein 2002a; Tanamachi et al. 2012; Wurman and Kosiba 2013). In other cases, cyclonicanticyclonic tornado pairs have been documented (e.g., Wurman and Kosiba 2013). In addition, "satellite" tornadoes are sometimes observed; these tornadoes are often very close to large and violent tornadoes, are weaker than the parent tornadoes, and tend to have short (i.e., $<10 \mathrm{~min}$ ) life cycles (e.g., Speheger et al. 2002; Tanamachi et al. 2012; Bodine et al. 2013; Wurman and Kosiba 2013; Bluestein et al. 2014; Edwards 2014). 
Though a formal definition for satellite tornadoes has only recently been proposed (Edwards 2014), anecdotally they typically orbit the parent tornado within the same mesocyclone (e.g., Speheger et al. 2002).

In one of the instances of simultaneous tornadoes in the El Reno-Piedmont supercell, the tornadic vortex signature $^{1}$ (TVS; Brown et al. 1978) associated with the second cyclonic tornado merged ${ }^{2}$ with the TVS associated with the ERPT (French et al. 2014). Based on the large number of visual sightings of tornadoes from the past 40 years and the increasing amount of highresolution radar data of tornadic supercells, the occurrence of merging tornadoes in a supercell appears to be a rare event. Excluding tornadic subvortices, the authors are aware of only two other supercell cases discussed in the literature in which simultaneous cyclonic tornadoes possibly merged: the interaction/merger of two tornadoes near Midway, Indiana, during the Palm Sunday Outbreak of 11 April 1965 (Fujita et al. 1970) and the interaction/merger of the Hesston and Goessel, Kansas, tornadoes on 13 March 1990 (Fujita 1992; Davies et al. 1994). ${ }^{3}$

In the Midway case (Fujita et al. 1970), a series of photographs and a damage survey [cf. Figs. 22-24 and 4647 in Fujita et al. (1970)] were used to conclude that a single tornado split into two tornadoes, which briefly rotated around each other before "reorganizing" into one tornado again. The splitting and reorganization process was estimated to take less than $1 \mathrm{~min}$. One photograph in the aforementioned short series shows a near-surface gap

\footnotetext{
${ }^{1}$ The TVS is viewed herein as approximately representative of the tornado, as in past studies of the ERPT (French et al. 2013, 2014). We expect that the true intensity (size) of the tornado is likely to be greater (less) than that of the TVS (e.g., Brown et al. 1978; Wood and Brown 1997).

${ }^{2}$ For the purposes of this study, and based on the results of 2D simulations of Rankine vortices (e.g., Waugh 1992), we define a tornado merger as occurring when two tornadoes interact such that neither persists individually after the interaction begins and the resulting tornado contains vertical vorticity from both original tornadoes. For this case, better visual observations or radar observations with greater spatial resolution in addition to vorticity trajectories would be necessary to state definitively that the tornadoes merged. Instead, we use visual images of two funnels interacting and radar data showing the concatenation of two TVSs as strong evidence that the tornadoes merged. Herein, we use "tornado merger" to describe the interaction in this case, but acknowledge that we cannot prove absolutely that the tornadoes merged in accordance with this definition.

${ }^{3}$ In Dowell and Bluestein (2002b), the authors discuss the merger of two vorticity maxima in a supercell on 8 June 1995 during the Verification of the Origins of Rotation in Tornadoes Experiment (VORTEX; Rasmussen et al. 1994), at least one of which was associated with a tornado. Later in the same storm, a tornado merger was observed by VORTEX teams near Allison, Texas (Grazulis 1997).
}

between two condensation funnels (Fig. 1a) and the damage survey shows the second tornado becoming larger than the original tornado (Fig. 1b). However, because of the very short amount of time in which separate tornadoes were observed and the short distance between them, it is possible, if not probable, that the two condensation funnels resulted from a single, two-celled, multiple vortex tornado (Ward 1972).

There is considerably more evidence to support the merger of the Hesston and Goessel tornadoes. Fujita (1992) asserts, based on a damage survey, that the two tornadoes rotated around a common point and that the track of the original tornado (Hesston) deviated such that it rotated around the second tornado (Goessel) as it was dissipating. Based on the survey (Fig. 1c), the Goessel tornado formed about $1.5 \mathrm{~km}$ to the rear of the weakening Hesston tornado and moved northeast, eventually interacting with the Hesston tornado as the Hesston tornado began to move more toward the north-northeast. After the interaction and possible merger, the resulting tornado was long lived and both larger and more intense [Fujita scale (F5)] than either of the individual tornadoes in the minutes preceding the interaction. In Davies et al. (1994), the merger of the tornadoes is not explicitly discussed; instead, the two tornadoes are said only to interact. However, the interaction of the two tornadoes was captured on film ${ }^{4}$ within which it can be seen that as the Hesston tornado condensation funnel dissipated, it was within or very close to the main circulation of the Goessel tornado, and may have met our criteria for tornado merger. The National Weather Service Weather Forecast Office in Wichita also states that the two tornadoes merged (http://www.crh.noaa.gov/ict/?n=hesston). What constitutes a tornado merger is not defined in any of the previous studies, but it is reasonable to assume that the use of the term implies an evolution similar to that described in this study.

In French et al. (2014), the authors investigated, among other topics, the steadiness of TVS intensity in time and height for the ERPT using data from a mobile, phasedarray Doppler radar - the Mobile Weather Radar, 2005 X-band, Phased Array (MWR-05XP; Bluestein et al. 2010). In radial velocity data from the MWR-05XP, it was shown that one TVS rotated around and then appeared to merge with another TVS [see Fig. 17a in French et al. (2014)]. We believe these data are evidence of the first published documentation of a temporally resolved tornado merger in volumetric mobile Doppler radar data. The strengthening and widening of the ERPT following

\footnotetext{
${ }^{4}$ As of the time of publication, the video was available online at http://www.youtube.com/watch?v=PslT4_HwPiM.
} 
(a)

(b)

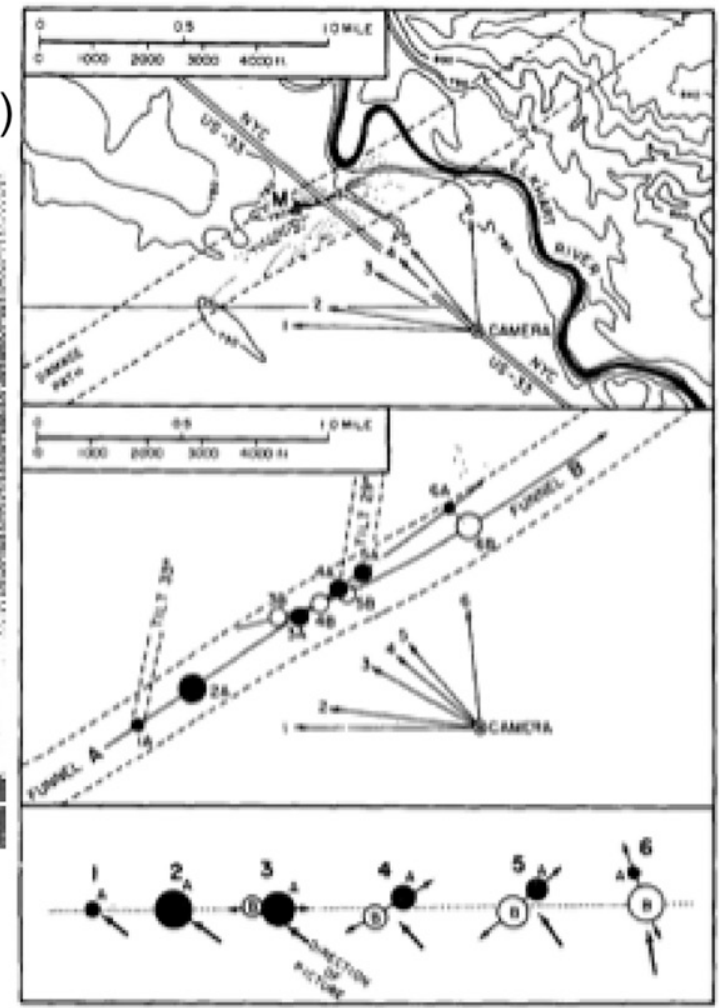

(c)
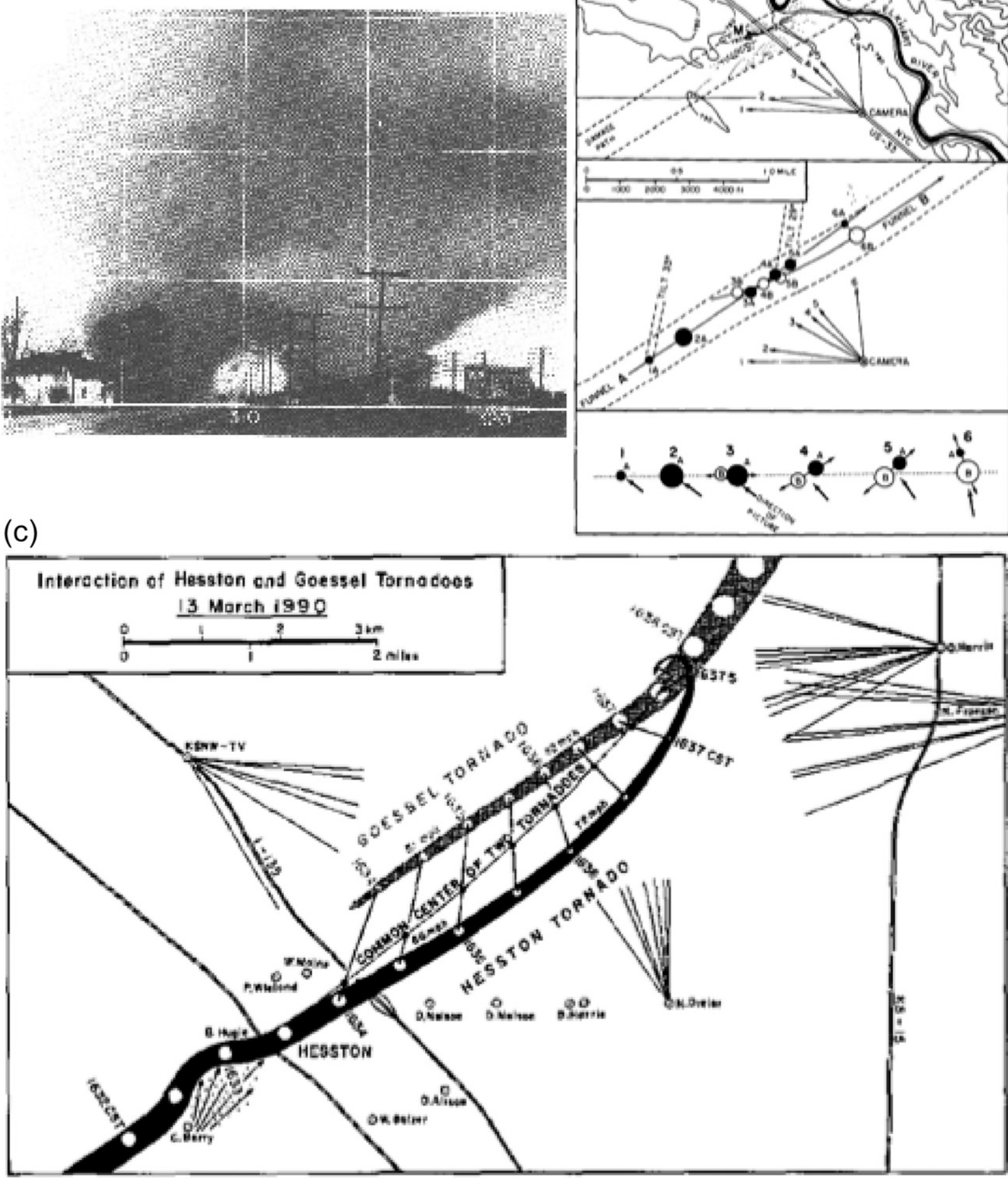

FIG. 1. (a) One of a series of six pictures of the Midway tornado on 11 Apr 1965, originally taken by P. Huffman of the Elkhart Truth. (b) (top) Part of the damage path of the Midway tornado, including at the time of the picture in (a). (b) (bottom) A schematic of the locations of the two tornadoes (black and white circles) along the damage path. (c) Analysis of the damage paths and interaction between the Hesston and Goessel tornadoes on 13 Mar 1990 from T. Fujita. Both (a) and (b) are adapted from Fujita et al. (1970) and (c) is from Davies et al. (1994). 
the merger and the lack of any previous Doppler radar studies of tornado mergers warrant a more detailed look at the tornado merger process. The focus of this paper is on the formation location of the second tornado, the volumetric evolution of the merger process, and the change in intensity and size of the ERPT during and after the merger. Section 2 provides a brief description of the data used in this study and section 3 presents data of TVS interaction and merger. The observations are discussed and put in the context of previous observations in section 4.

\section{Data}

\section{a. MWR-05XP}

On 24 May 2011, a team from the University of Oklahoma, participating in an annual spring field experiment, obtained data of the EF5 ERPT with two mobile Doppler radars: the aforementioned MWR-05XP and the Rapid, X-band Polarimetric radar (RaXPol; Pazmany et al. 2013). There were two MWR-05XP deployments on the supercell: one that began just prior to formation of the ERPT (French et al. 2013) and lasted from 2049:30 to 2056:04 UTC (tornadogenesis was estimated at 2050 UTC) and a second deployment that began $37 \mathrm{~min}$ later and lasted from 2133:43 to 2203:00 UTC (hereafter all times in UTC). The TVS interaction and merger were observed at the beginning of the second deployment. A RaXPol deployment prior to the second MWR-05XP deployment was terminated several minutes before genesis of the second tornado (Houser et al. 2015), so data from the WSR-88D in Twin Lakes, Oklahoma (KTLX), a prototype polarimetric WSR-88D in Norman, Oklahoma (KOUN), and the multifunction phased array radar (MPAR; Zrnić et al. 2007) are used to document the formation of the second tornado. A timeline of the estimated tornado times and volume scan start times of the pertinent radars is shown in Fig. 2.

The MWR-05XP is an X-band system that utilizes electronic scanning in elevation and in azimuth. The radar has high temporal resolution but a relatively broad beamwidth of $1.8^{\circ}\left(2.0^{\circ}\right)$ in azimuth (elevation). During the El Reno-Piedmont deployment, the MWR-05XP collected $90^{\circ}$ sector scans at 26 elevation angles from $1^{\circ}$ to $40^{\circ}$ every $11 \mathrm{~s}$. Despite a presumed level site for the second deployment [cf. Fig. 1e in French et al. (2014)], the lack of a functioning hydraulic leveling system on the MWR-05XP introduced the possibility of errors in TVS position, particularly in height. As a result of this uncertainty, an emphasis here is placed on qualitative height levels rather than exact heights. For details about radar electronic scanning and other radar parameters, see Bluestein et al. (2010). For additional information about

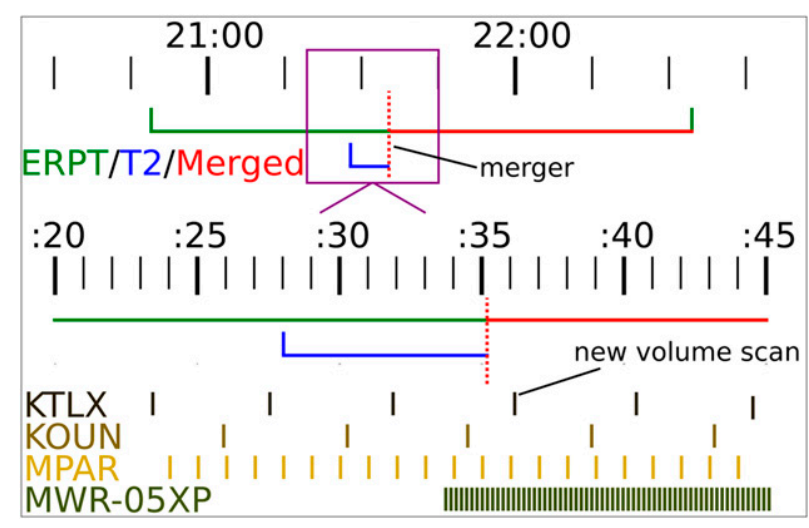

FIG. 2. (top) Timeline of the El Reno-Piedmont tornado (green), the second tornado (blue), and the merged tornado (red); tick marks every 15 min. (bottom) Zoomed-in timeline showing KTLX, KOUN, MPAR, and MWR-05XP volume scan start times at and around the time of the merger; timeline tick marks are every $1 \mathrm{~min}$. Formation time of T2 is estimated as 2128 UTC based on MPAR data. All times are in UTC, bottom times omit the 2100 UTC hour.

the El Reno-Piedmont supercell dataset, including estimated TVS position errors, see French et al. (2014).

The formation of the second tornado was observed by a suite of S-band radar (10-cm wavelength) systems: KTLX, KOUN, and MPAR. All of the S-band systems are located in the Oklahoma City area, within $50 \mathrm{~km}$ of El Reno. KTLX and KOUN have comparatively better spatial resolution than MPAR and KOUN is a dualpolarization system. ${ }^{5}$ The advantage of MPAR is its frequent volumetric updates (every $1 \mathrm{~min}$ ). Data from all three systems are used to examine the formation of the second tornado until the time when MWR-05XP data are available (Fig. 2).

\section{b. Azimuthal wind shear}

Three-dimensional regions of vertical rotation in the El Reno-Piedmont supercell were approximated using isosurfaces of objectively analyzed azimuthal wind shear. Azimuthal wind shear was calculated in MWR-05XP data as $\left(r^{-1}\right) \partial V_{r} / \partial \phi$, where $V_{r}$ is the radial velocity, $\phi$ is the azimuth, and $r$ the range for each gate considered. Azimuthal wind shear values were then interpolated to a Cartesian grid using the two-pass Barnes's objective analysis scheme (Barnes 1964) described by Majcen et al. (2008). Data were only interpolated to regions within $15 \mathrm{~km}$ of the MWR-05XP as this region encompasses the mesocyclone and merging tornadoes. Using a maximum range from the radar of $15 \mathrm{~km}$ plus the radius of influence for the Barnes's analysis [calculated as the square root of

\footnotetext{
${ }^{5}$ Both KTLX and KOUN currently have dual-polarization capabilities, but only KOUN had them at the time of the observations.
} 


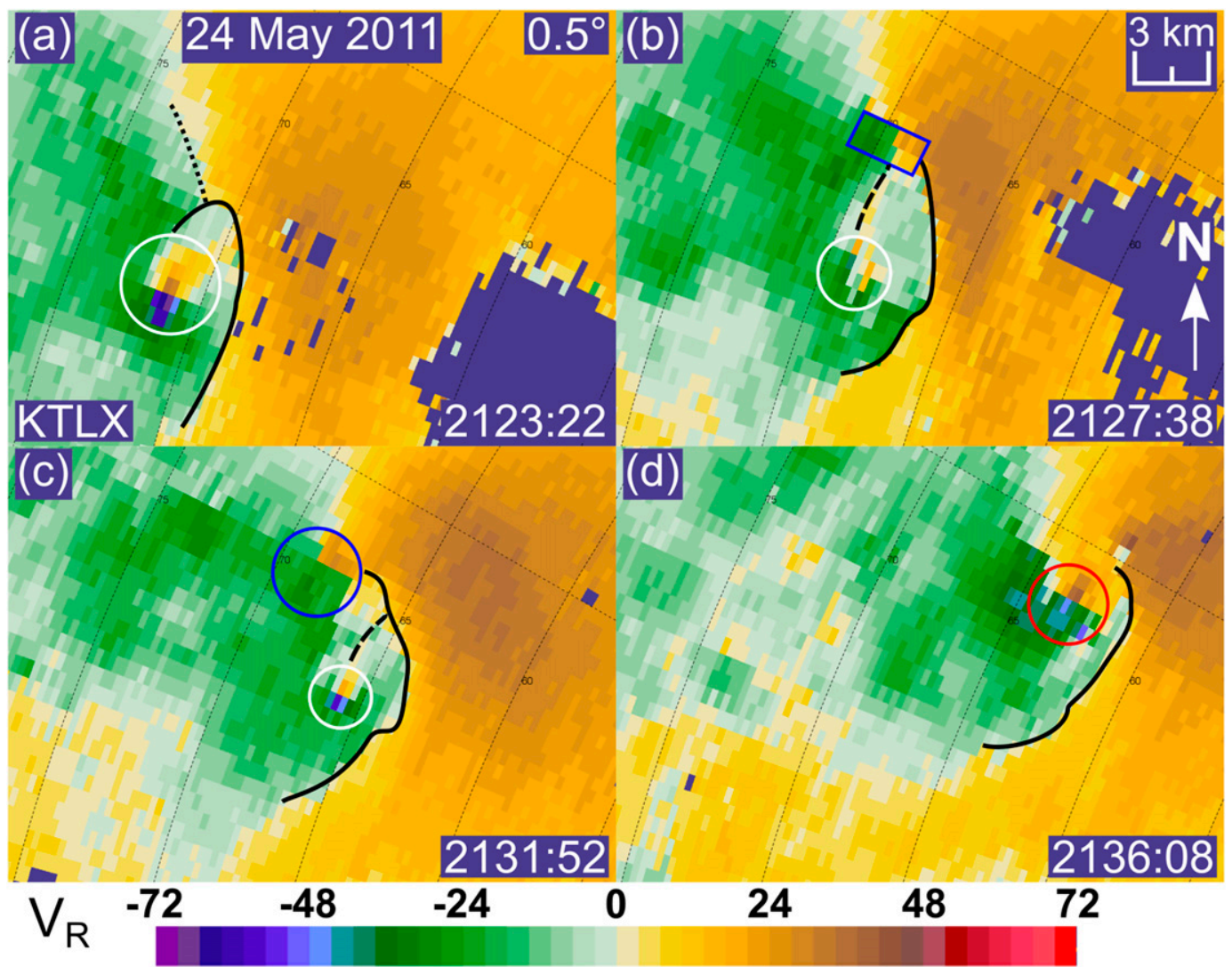

FIG. 3. Plan position indicator displays (PPIs) of radial velocity $\left(\mathrm{m} \mathrm{s}^{-1}\right.$ ) from KTLX on 24 May 2011 at (a) 2123:22, (b) 2127:38, (c) 2131:52, and (d) 2136:08 UTC. The white (blue) circle(s) enclose TVSs associated with the El RenoPiedmont (second) tornado; in (d) the TVS resulting from the merger is outlined by a red circle. The solid black lines mark areas of radial convergence along the leading edge of the rear-flank gust front. The dotted black line in (a) is an area of weak radial convergence north of the rear-flank gust front and the blue rectangle in (b) is an area of radial convergence. The dashed black lines in (b) and (c) mark areas of weaker radial convergence leading to the second TVS. Range rings are every $5 \mathrm{~km}$. The images are centered at the same location. The approximate heights of the center of the radar beams at the locations of the TVSs enclosed by white or red circles are (a) 630, (b) 590, (c) 575, and (d) $550 \mathrm{~m}$ ARL.

5 times the Barnes smoothing parameter $(\kappa)]$ resulted in a coarsest grid spacing $(\mu)$ of $578 \mathrm{~m}$, and a $\kappa$ value [calculated as $\left.(1.33 \mu)^{2}\right]$ of 0.591 for a grid with $200-\mathrm{m}$ spacing.

\section{The EI Reno-Piedmont tornado merger}

\section{a. Formation of the second tornado}

In MWR-05XP data shown in French et al. (2014), the two tornadoes are displaced from each other in the northsouth direction. The authors posited that the northern of the two tornadoes was the ERPT based on (i) the southern tornado's erratic path around the northern tornado and (ii) the normal progression of the cyclic tornadogenesis process in which the second tornado (hereafter T2) forms southeast of the original tornado (Burgess et al. 1982; Dowell and Bluestein 2002a). In KTLX data (Fig. 3), at 2123 (Fig. 3a), there is one large
TVS with strong radial velocities (as high as $67 \mathrm{~m} \mathrm{~s}^{-1}$ ); based on all available information, this TVS is associated with the ERPT. In the next volume scan, at 2128 (Fig. 3b), there is still one main area of rotation, but radial convergence has increased northwest of another well-defined area of convergence likely associated with the rear-flank gust front (RFGF). At 2132 (Fig. 3c), there are two TVSs; the second one develops in the area of radial convergence present in the previous volume. At 2136 (Fig. 3d), there is only one TVS again, though it is now larger and contains stronger radial velocities than in the previous two volumes. The KTLX data are strong evidence that the southern tornado was the ERPT instead of the northern tornado.

To investigate further the formation of $\mathrm{T} 2$, KOUN radial velocity and polarimetric data were compared to the location of the surveyed damage path at $\sim 900 \mathrm{~m}$ above radar level (ARL; Fig. 4). Cross-correlation 

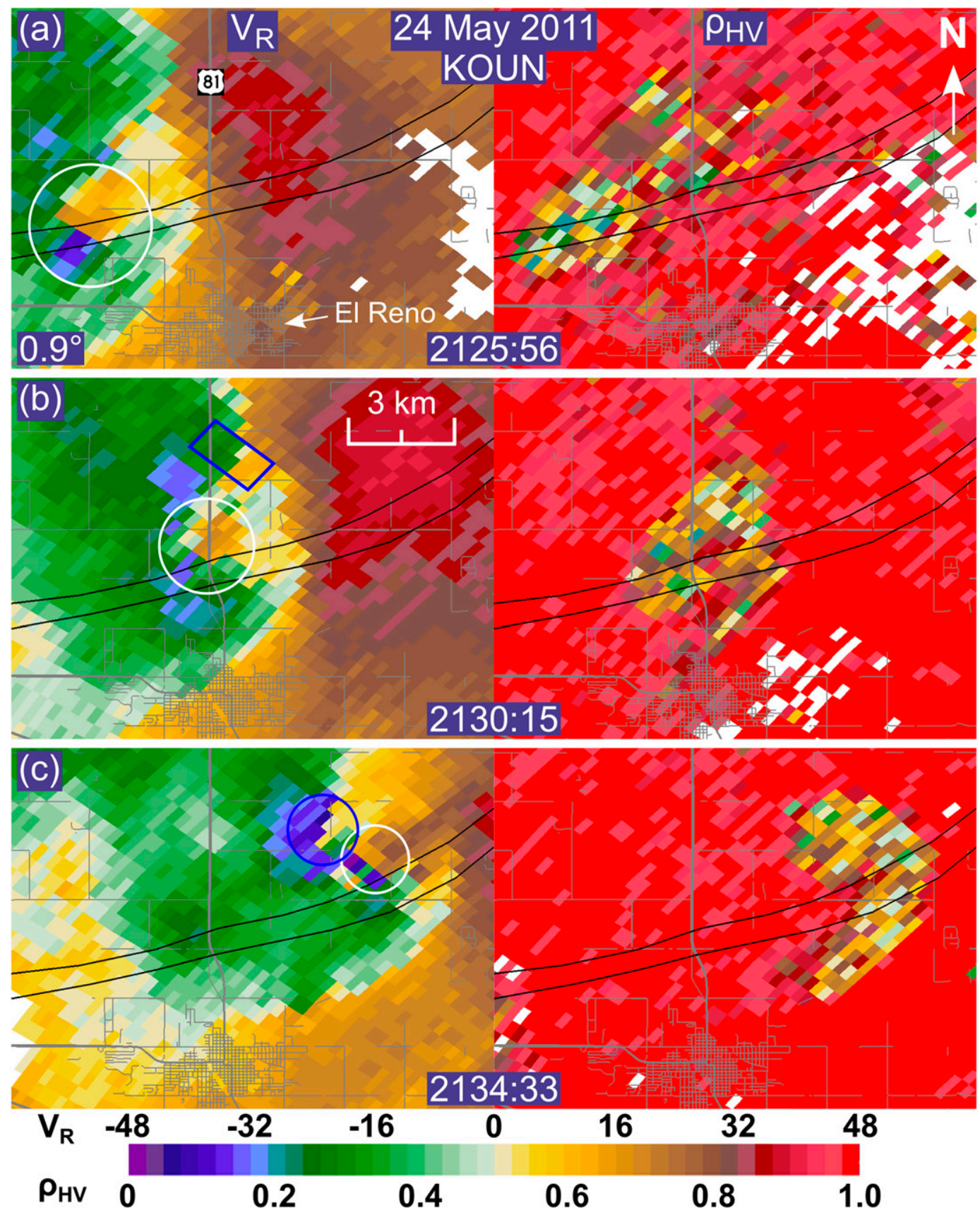

FIG. 4. PPIs of (left) radial velocity $\left(\mathrm{m} \mathrm{s}^{-1}\right)$ and (right) copolar cross-correlation coefficient from KOUN on 24 May 2011 at (a) 2125:56, (b) 2130:15, and (c) 2134:33 UTC. The black lines outline the surveyed El Reno-Piedmont tornado damage path and gray lines mark state and county roads. The white and blue circle(s) and the blue rectangle outline the same features as in Fig. 3. In (b), there is both strong radial convergence and cyclonic shear at the location of the rectangle, but only the former is outlined for convenience. The images are centered at the same location. The locations of El Reno and Highway 81 are labeled in (a). Note the different radial velocity scale from that used in Fig. 3. The approximate heights of the centers of the radar beams at the locations of the TVSs enclosed by white circles are (a) 940, (b) 925, and (c) $910 \mathrm{~m}$ ARL.

coefficient $\left(\rho_{\mathrm{HV}}\right)$ data were inspected for a tornadic debris signature (TDS; Ryzhkov et al. 2002, 2005; Bluestein et al. 2007), where an area of low values $\left(\rho_{\mathrm{HV}}<0.8\right)$ collocated with a TVS indicates debris caused by tornadic winds. At 2126, similar to that shown in KTLX data, there is one TVS with a corresponding TDS that overlays directly on to the surveyed ERPT damage path. At 2130 (Fig. 4b), the previously identified 
area of radial convergence is located about $4 \mathrm{~km}$ north of the surveyed damage path, while the TVS and associated TDS are roughly overlaid with the damage path. By 2134 (Fig. 4c), there are two TVSs: the northern TVS is located north of the damage path and the southern TVS overlaps with the northern edge of the damage path. The TDS is now larger and incorporates both TVSs.

In addition to radar data, photographs of the supercell also provide evidence of two tornadoes that interact (Fig. 5). Two distinct lowerings are annotated in a photograph looking southwest toward the El Reno area at 2133 (Fig. 5a). The lowering to the right is easy to identify as a funnel cloud; the area to the left appears nebulous, likely owing to the presence of heavy precipitation at the location of the ERPT. At 2134 (Fig. 5b), there are still two lowerings, but they are now closer to each other and more difficult to differentiate. By 2137 (Fig. 5c), there is only one tornado, which appears larger in size than the ERPT from 2133 to 2134. Also, video from just north of El Reno looking north at 2130 (not shown) first shows a large dark lowering moving from west to east and causing power flashes east of Highway 81. Shortly after, a tornado is seen west of Highway 81 also moving eastward. This progression is consistent with $\mathrm{T} 2$, or its larger-scale predecessor, crossing Highway 81 before the ERPT. Radial velocity data, polarimetric radar data, still photographs, and video all provide evidence that there were two tornadoes that interacted, and that the southern (northern) tornado was the ERPT (T2). The assumptions made in French et al. (2014) regarding the latter point are in error.

The temporal evolution of $\mathrm{T} 2$ formation was best captured by the MPAR (Fig. 6). The formation of the second TVS in an area of radial convergence and the subsequent strengthening of the second TVS occurs over a period of $\sim 4 \mathrm{~min}$. The aforementioned radial convergence rapidly increases in the 2 -min period from 2125 to 2127 (Figs. 6a-c). In the subsequent minute (Fig. 6d), the area of cyclonic convergence transitions to an easily identifiable TVS that is associated with T2. During this same time period, ERPT TVS $\Delta V$ at the lowest-observed level decreases greatly, from 106 to $48 \mathrm{~m} \mathrm{~s}^{-1}$. To the authors' knowledge, it is extremely rare for a second well-defined TVS to be identified in WSR-88D and spatially coarse MPAR data so close to an existing TVS and within the same mesocyclonescale area of rotation.

Data from KOUN and MPAR also are used to examine the supercell at midlevels (2-4 km ARL) during the formation of T2. Of particular interest is the evolution of mesocyclones/updrafts in the storm during the formation of T2. No data suitable for dual-Doppler analyses were obtained; however, in KTLX and KOUN single-Doppler radial velocity data (not shown), an elongated area of cyclonic rotation with two individual maxima was identified. The locations of minima in $\rho_{\mathrm{HV}}$, and columns of differential radar reflectivity factor, $Z_{\mathrm{DR}}$ (e.g., Illingworth et al. 1987; Conway and Zrnić 1993), were used to estimate updraft positions in the El RenoPiedmont supercell (Fig. 7). Storm updrafts are typically collocated with $\rho_{\mathrm{HV}}$ minima aloft (e.g., Ryzhkov et al. 2005) and, similarly, collocated or slightly offset from $Z_{\mathrm{DR}}$ columns in supercells (e.g., Kumjian and Ryzhkov 2008; Snyder et al. 2013; Kumjian et al. 2014). During the weakening of the ERPT and formation of T2, there is one main $Z_{\mathrm{DR}}$ column indicating the main midlevel updraft. A secondary area of enhanced $Z_{\mathrm{DR}}$ to the south of the primary area is associated with another updraft along the southern part of the RFGF (Fig. 7a), which becomes closer to and merges with, the main updraft (Figs. 7b,c). The location of the TVS associated with the ERPT, both near the surface and aloft, becomes increasingly displaced from the main $Z_{\mathrm{DR}}$ column through 2128 UTC (Figs. 7a-c). Subsequently, the second TVS forms (Fig. 7d) collocated with this same portion of the $Z_{\mathrm{DR}}$ column. It is also seen from both KOUN $\rho_{\mathrm{HV}}$ minima and 3D isosurfaces of azimuthal shear estimated from MPAR single-Doppler radial velocities (not shown) that there is only one large area of mesocyclonescale rotation.

\section{b. Merger of the two tornadoes}

Only data from the MWR-05XP were used to document the merger because data from KTLX and KOUN are too temporally coarse (volume scans every 4$4.5 \mathrm{~min}$ ) to do so (e.g., Figs. 2 and 3c,d). MPAR data do show two distinct TVSs before the merger, but as the two TVSs near each other, they become indistinguishable and their interaction is not spatially resolved well (not shown). Data from the MWR-05XP begin at 2133: 43, after the second TVS is identified in WSR-88D data. The radar's volumetric update time of $11 \mathrm{~s}$ and its distance to the two TVSs $(<15 \mathrm{~km})$ allows for the interaction of the two TVSs to be documented in the 2 min leading up to and through the merger (Figs. 8 and 9). For the purposes of this study, the TVSs are said to have merged when there are no longer two distinct radial velocity couplet maxima, and there is no evidence that one of the original TVSs dissipated during the interaction.

At the start of the second MWR-05XP deployment, two TVSs are identifiable in data from $1.0^{\circ}$ to $19.0^{\circ} \mathrm{el}-$ evation angle (heights of $0.1-4 \mathrm{~km}$ ARL; e.g., Figs. $8 \mathrm{a}$ and 9a). Over the first $\sim 1$ min of the deployment in data from the lowest-observed level, the El Reno-Piedmont 


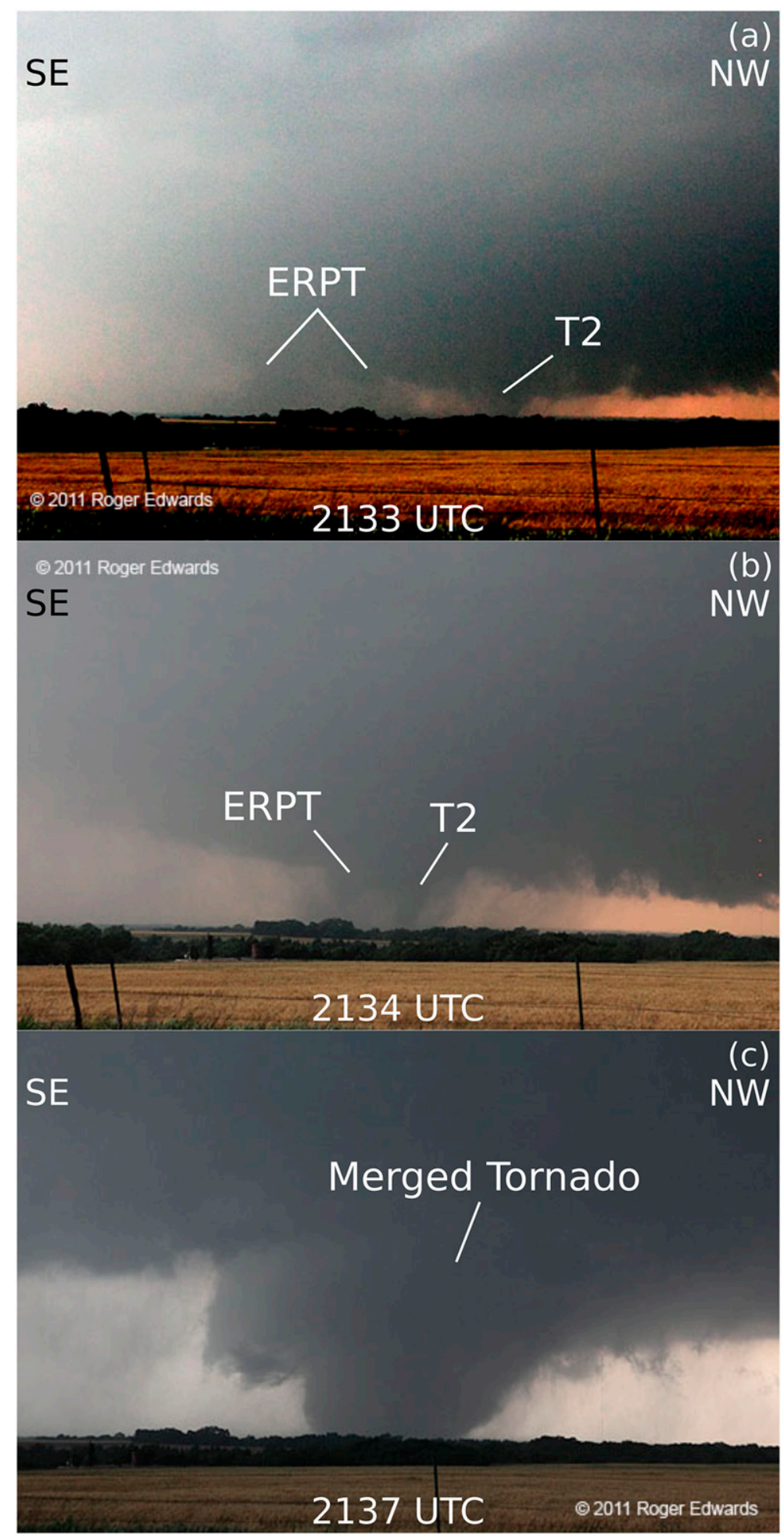

FIG. 5. A series of photographs showing the interaction and possible merger of the El Reno-Piedmont tornado and the second tornado. The photographs were taken $\sim 1$ mile south of OK-3 on Cimarron Rd., looking southwest toward El Reno at (a) 2133, (b) 2134, and (c) 2137 UTC 24 May 2011. The locations of the tornadoes are annotated. The image in (a) is enhanced to better show the two distinct lowerings. The photographs are zoomed in to different degrees so the spatial area represented in each is different. (All photographs are courtesy of R. Edwards.) 


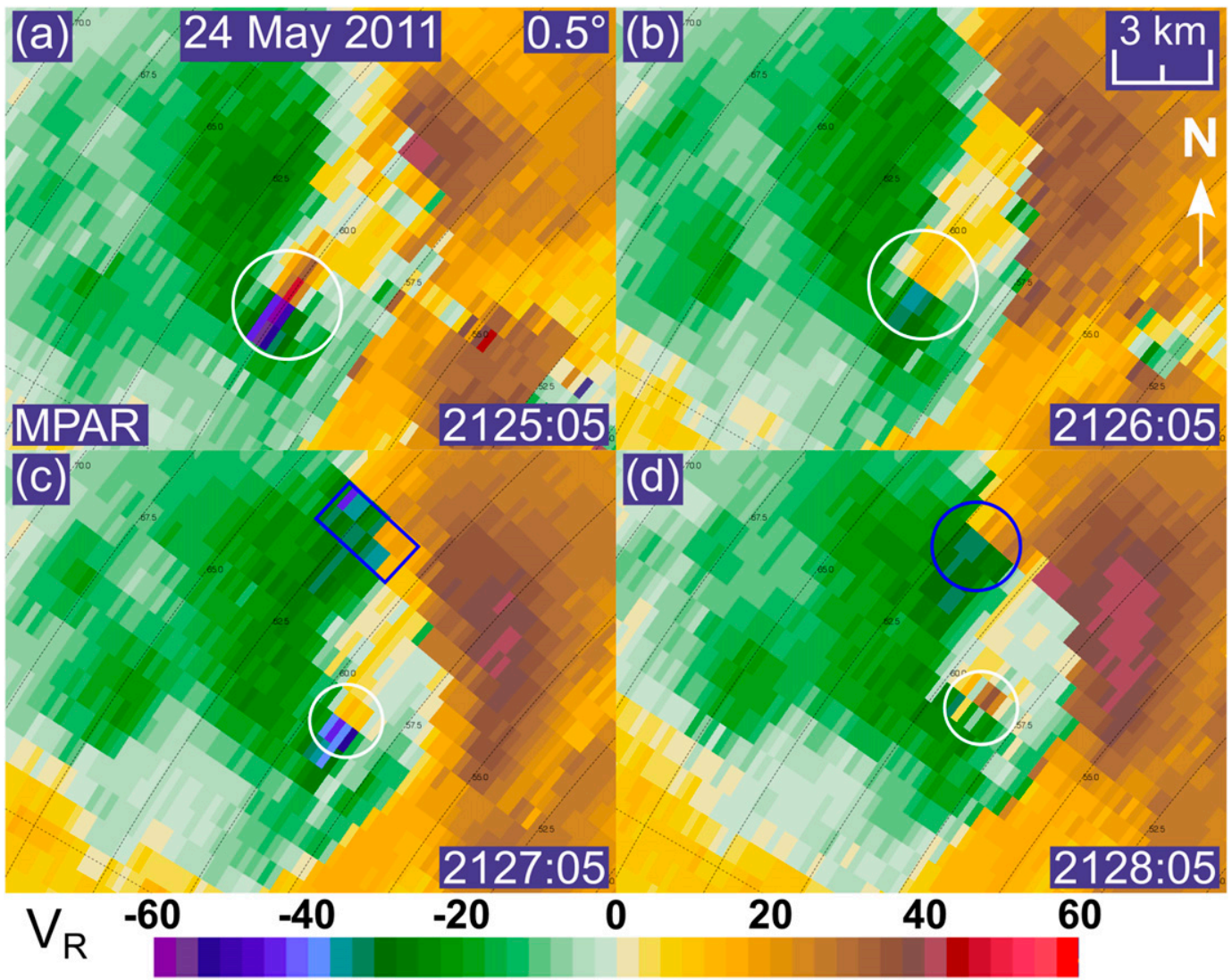

FIG. 6. As in Fig. 3, but from the MPAR at (a) 2125:05, (b) 2126:05, (c) 2127:05, and (d) 2128:05 UTC. Range rings are every $2.5 \mathrm{~km}$. The approximate heights of the centers of the radar beams at the location of the TVSs enclosed by white circles are $500 \mathrm{~m}$ ARL.

TVS (white circle) travels northward toward the newly developed second TVS (blue circle), which translates slowly east (Figs. 8a-e). Between 2134:38 and 2135:00, the El Reno-Piedmont TVS continues to move north and the second TVS moves south and then east-southeast as the distance between the two TVSs decreases (Figs. 8f-h); in animation (see Fig. S1 in the online supplemental material), the TVSs engage in counterclockwise rotation around a common center (Fujiwhara 1931). Beginning at 2135:11 and continuing through the end of the observational period, there is a single TVS that is wider and more intense (see section 3c) than either of the individual TVSs (Figs. 8i-1).

The progression of the merger follows a similar path aloft at a height of $\sim 2.5 \mathrm{~km}$ ARL (Fig. 9). In the first minute of data collection (Figs. 9a-f), the El RenoPiedmont TVS moves north and then northwest while the second TVS begins to move southeast as they approach each other. However, aloft the two TVSs rotate $\sim 270^{\circ}$ around each other while maintaining separate maxima in $\Delta V$ (Figs. 9g-k). By 2135:43 (Fig. 91), the two TVSs can no longer be distinguished and, based on our definition, have merged. The merger aloft is completed within three scans (32s) of that in data near the surface (Fig. 8i), though given that the tornadoes are not resolved well, the brief period of time between the merger near the surface and aloft may not be meaningful. The paths of the two TVSs and their rotation around each other are shown in Fig. 10 for three different height levels. The location of the TVS was estimated by using the location of the max $\Delta V$ in the TVS. Aloft (e.g., Figs. 10b-e), the two TVSs complete at least three quarters of a full rotation around each other. In addition, the storm-relative motion vectors of the TVSs are shown (top left of Fig. 10a); the initial TVSs converge toward each other, however, the merged TVS moves in a direction very similar to that of the storm.

The merger is also examined using 3D isosurfaces of azimuthal wind shear overlaid on the approximate center of the TVSs in MWR-05XP volumes looking from the south (Fig. 11). From this perspective and all others (not shown), there is only one consistent area of broad azimuthal wind shear leading up to the merger. As shown previously, the TVSs move closer to each other as 


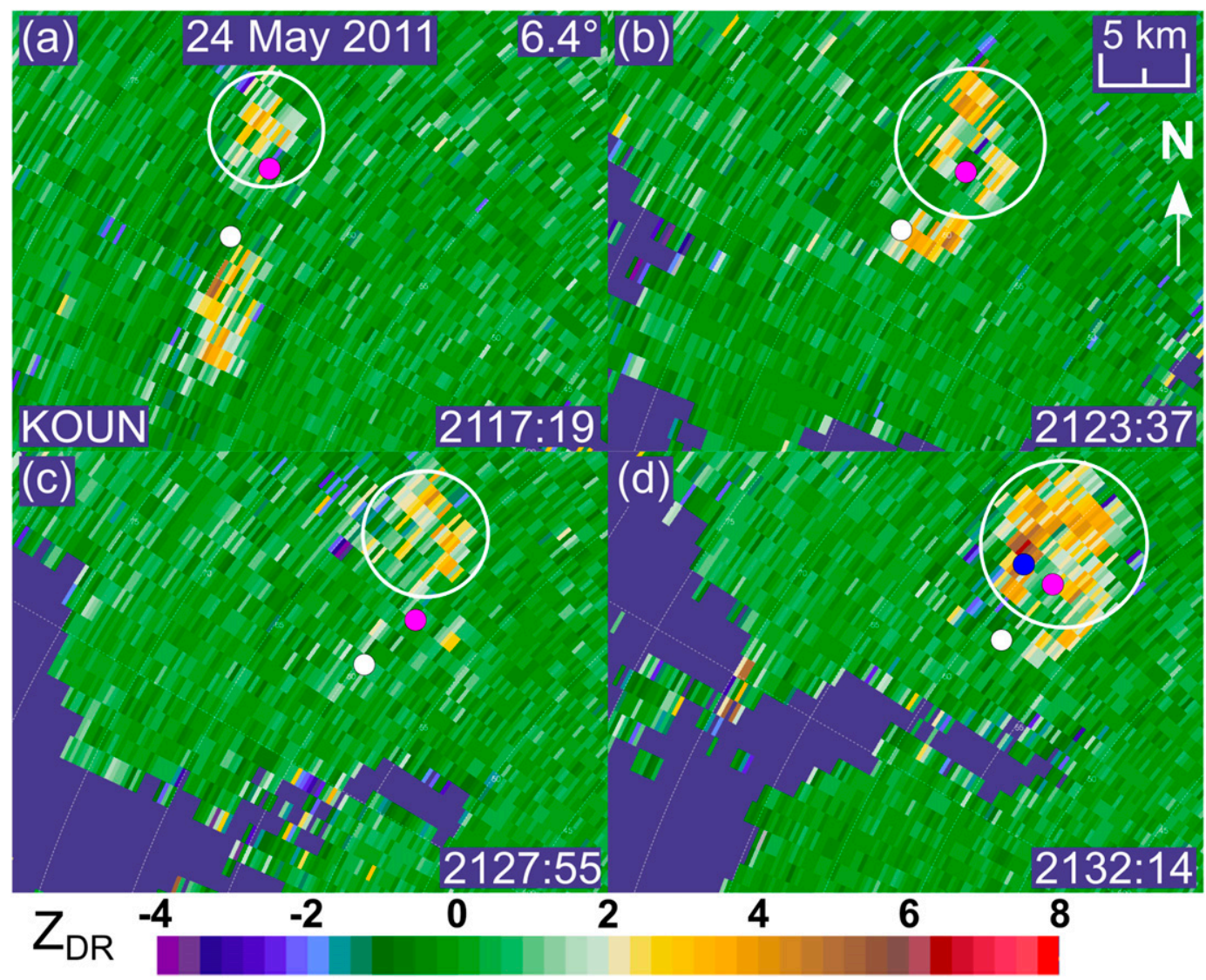

FIG. 7. PPIs of $Z_{\mathrm{DR}}$ (dB) from KOUN on 24 May 2011 at (a) 2117:19, (b) 2123:37, (c) 2127:55, and (d) 2132:14 UTC. The main areas of enhanced $Z_{\mathrm{DR}}$ are outlined by white circles. The approximate locations of the TVSs associated with the El Reno-Piedmont tornado at $6.4^{\circ}$ and $0.5^{\circ}$ elevation angle $(\sim 7$ and $\sim 0.5 \mathrm{~km}$ ARL $)$ are marked by fuchsia and white dots, respectively; the location of the second TVS at $0.5^{\circ}$ elevation angle is marked by a blue dot in (d). Range rings are every $5 \mathrm{~km}$. The approximate height of the center of the radar beam at the location of the main $Z_{\mathrm{DR}}$ column is $7 \mathrm{~km}$ ARL.

they move north and east, respectively (Figs. 11a-d). Based on the radar data, the merger of the TVSs takes place first at the lowest-observed level (Fig. 11e) and then at higher levels (Figs. 11f-h). Notably, the TVSs wrap around each other in a helical manner (i.e., the TVSs are offset from one another in different directions at different heights; Figs. 11e-g; see Fig. S2 in the online supplemental material). Additionally, a rapid increase in azimuthal wind shear is apparent during and immediately following the merger (Figs. 11c-g). Also, both TVSs are tilted prominently toward the north prior to and during the merger (not shown), consistent with previous observations of tornado tilt (e.g., Wurman and Gill 2000; Alexander and Wurman 2005; Tanamachi et al. 2012; French et al. 2014).

\section{c. Posttornado-merger observations}

French et al. (2014) studied the merger in the context of large changes in TVS intensity over short periods of time. Prior to the merger, the intensity of both the El
Reno-Piedmont TVS and the second TVS is relatively steady with $\Delta V$ of $65-75 \mathrm{~m} \mathrm{~s}^{-1}$ at the lowest-observed level. However, after the merger, there is an immediate and consistent increase in TVS $\Delta V$ to over $100 \mathrm{~m} \mathrm{~s}^{-1}$ (Fig. 12a). Based on the results of Rankine vortex merger simulations [e.g., Waugh (1992); see section 4 of this paper], the increase in intensity at or just after the time of the merger is circumstantial evidence of a cause and effect relationship. The intensity increase is not short lived as $\Delta V$ of the merged tornado stays near or above $100 \mathrm{~m} \mathrm{~s}^{-1}$ for the next $5+\min$. Also, the increase in TVS intensity is not relegated to the lowest-observed level; at all observed levels, there is an increase in TVS $\Delta V$ that lasts several minutes (e.g., Fig. 12b). There is no evidence of one TVS being "dominant"; at all levels the two TVSs have similar intensities leading up to the merger.

In addition to the increase in intensity, the merged TVS is wider. To estimate the increase in size of the TVSs (the tornado is not spatially resolved well in MWR-05XP data), the number of range-consecutive 


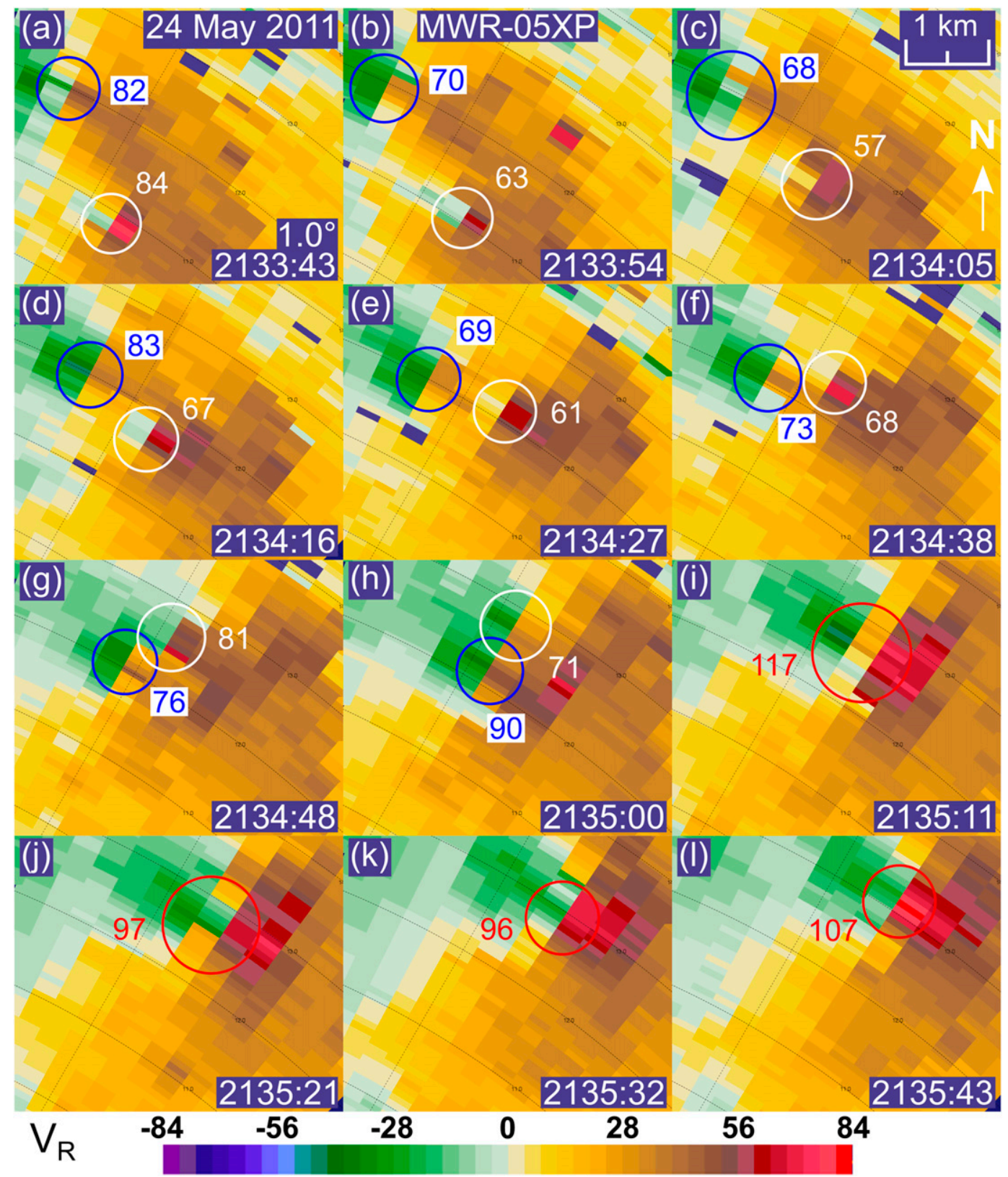

FIG. 8. PPIs of radial velocity $\left(\mathrm{m} \mathrm{s}^{-1}\right)$ from the MWR-05XP at $1.0^{\circ}$ elevation angle on 24 May 2011 at (a) 2133:43, (b) 2133:54, (c) 2134:05, (d) 2134:16, (e) 2134:27, (f) 2134:38, (g) 2134:48, (h) 2135:00, (i) 2135:11, (j) 2135:21, (k) 2135:32, and (1) 2135:43 UTC. The circles outline the same features as in Fig. 3. The color-coded numbers are the maximum $\Delta V$ in the corresponding TVS. Range rings are every $1 \mathrm{~km}$. All images are centered at the same location. The approximate heights of the centers of the radar beams at the locations of the outlined TVSs vary but generally are $\sim 100 \mathrm{~m}$ ARL.

gates with $\Delta V$ beyond various threshold values are calculated for the two original TVSs and the merged TVS ${ }^{6}$

\footnotetext{
${ }^{6}$ It is possible that changes in the surrounding flow outside of the tornado could bias these calculations, so the focus should be on qualitative trends rather than exact values.
}

at the lowest-observed level (Fig. 13a) and at a height of $\sim 2 \mathrm{~km}$ ARL (Fig. 13b). At both levels, the individual TVSs remain similarly sized up until the approximate time of the merger. Once the merger occurs or shortly after, there is a large increase in the size of the resulting TVS. This increase in size does not result from two poorly resolved adjacent TVSs because the change in the TVS 


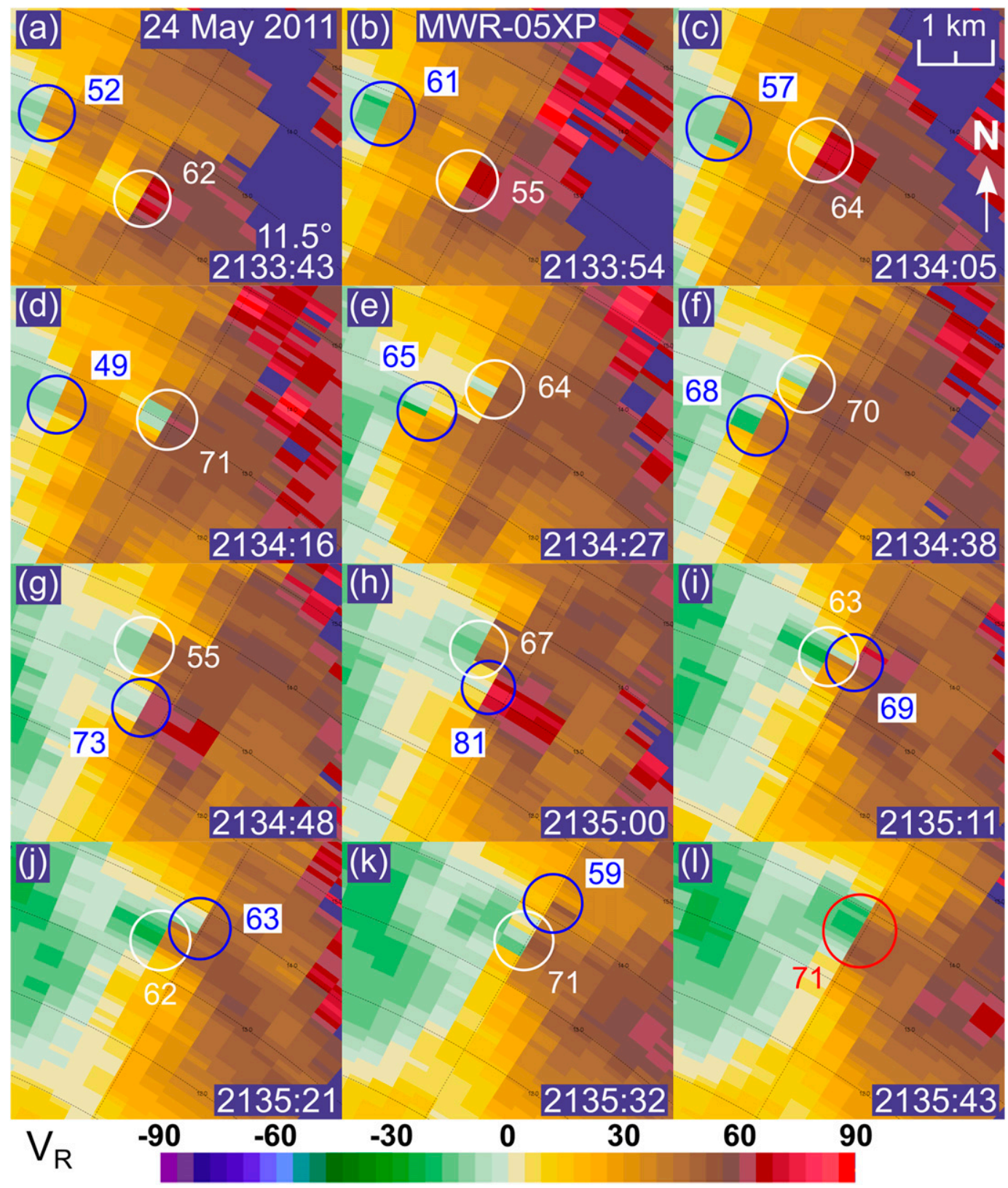

FIG. 9. As in Fig. 8, but at $11.5^{\circ}$ elevation angle. The approximate heights of the centers of the radar beams at the locations of the outlined TVSs vary but generally are $\sim 2600 \mathrm{~m}$ ARL. Note the different radial velocity scale from that in Fig. 8.

size is maintained and then increases in the minutes after the merger. In addition, calculating the number of gates with radial velocity magnitudes of $50 \mathrm{~m} \mathrm{~s}^{-1}$ or greater and lying within a $500 \mathrm{~m} \times 500 \mathrm{~m}$ box centered on the strongest positive and negative radial velocity magnitudes in the TVS yielded similar trends in TVS size (Fig. 13c). The latter plot illustrates that, in this case, the surface area being affected by very strong ground-relative winds likely increased after the merger despite there being one tornado instead of two. The trends shown in Fig. 13 are consistent with visual images of a larger tornado after the merger (e.g., Fig. 5c).

Before (after) the merger, the individual (merged) TVS(s) were moving away from the MWR-05XP. The increasing distance and, therefore, coarser radar azimuthal resolution, could lead to relative decreases in TVS intensity and relative increases in TVS size with time. Given the small changes in range during the MWR-05XP time series $(<2 \mathrm{~km})$, we believe these effects to be small. 
(a)

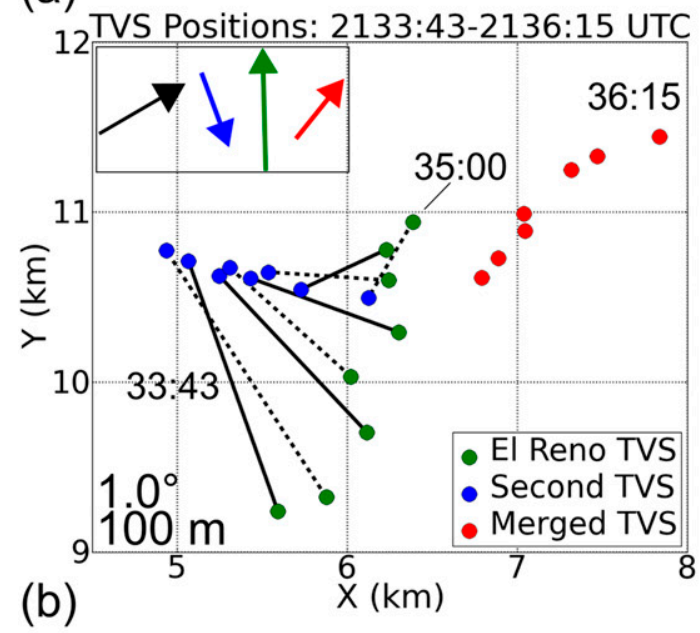

(d)
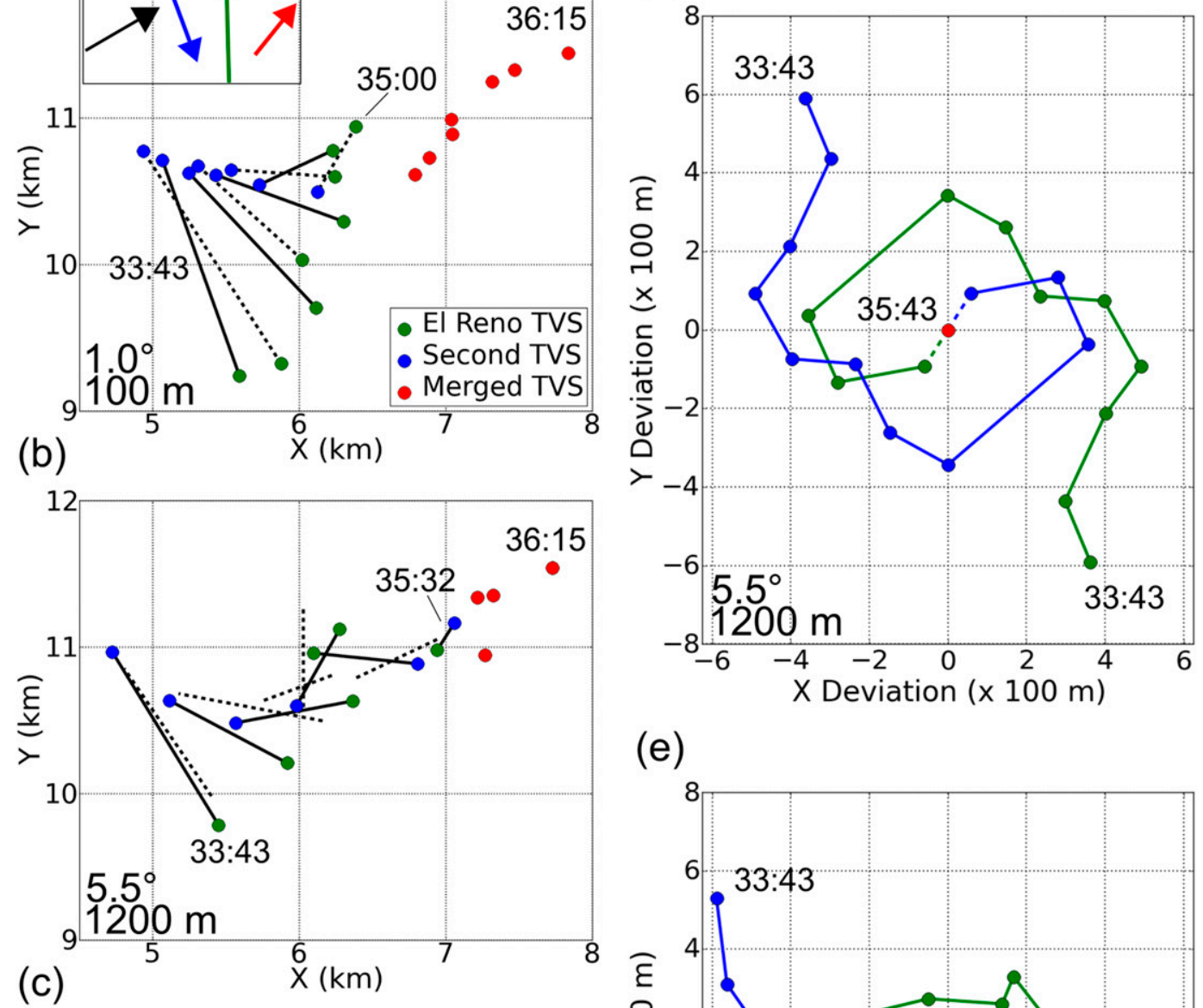

(e)
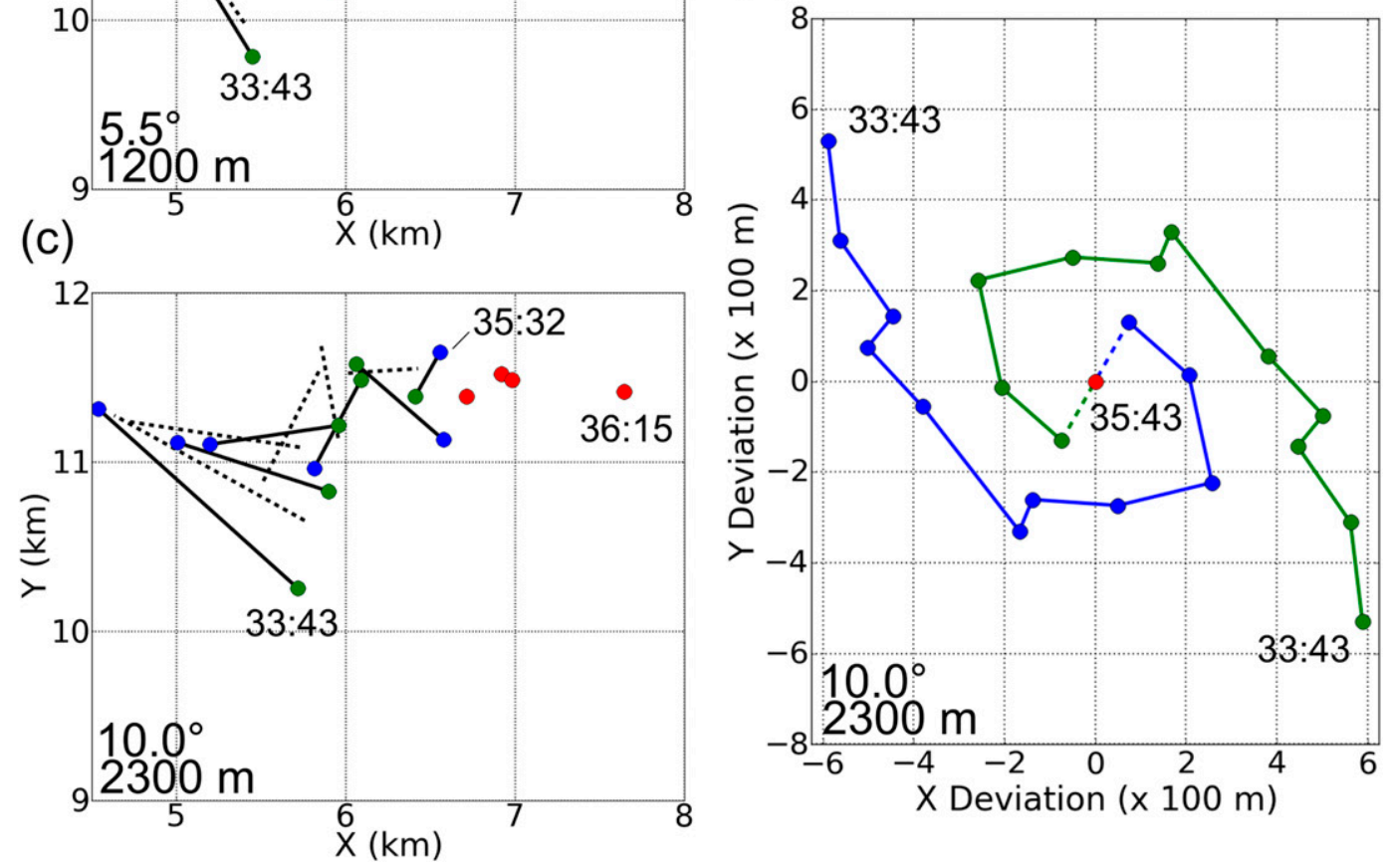

FIG. 10. Horizontal cross-sectional locations of the TVSs associated with the El Reno-Piedmont, second, and merged tornadoes on 24 May 2011 at (a) $1.0^{\circ}$; (b),(d) $5.5^{\circ}$; and (c),(e) $10.0^{\circ}$ elevation angle. (a)-(c) TVS position for each time with the locations of the El Reno-Piedmont and second TVSs connected by a black line. The black lines alternate between full and dashed lines chronologically. In (b) and (c) the locations of the El Reno-Piedmont and second TVSs are omitted at every other time for clarity. (d),(e) TVS position measured as the deviation from the midpoint of a line connecting the two TVSs at each time. The approximate height levels of the observations are shown in the bottom left. The first and last times, and for (a)-(c), the time directly preceding the merger, are marked. In the upper left of (a) appears, from left to right, the estimated storm motion vector as determined from KTLX data and the color-coded storm-relative TVS motion vectors. For (a)-(c), the origin is the location of the MWR-05XP (not shown). 

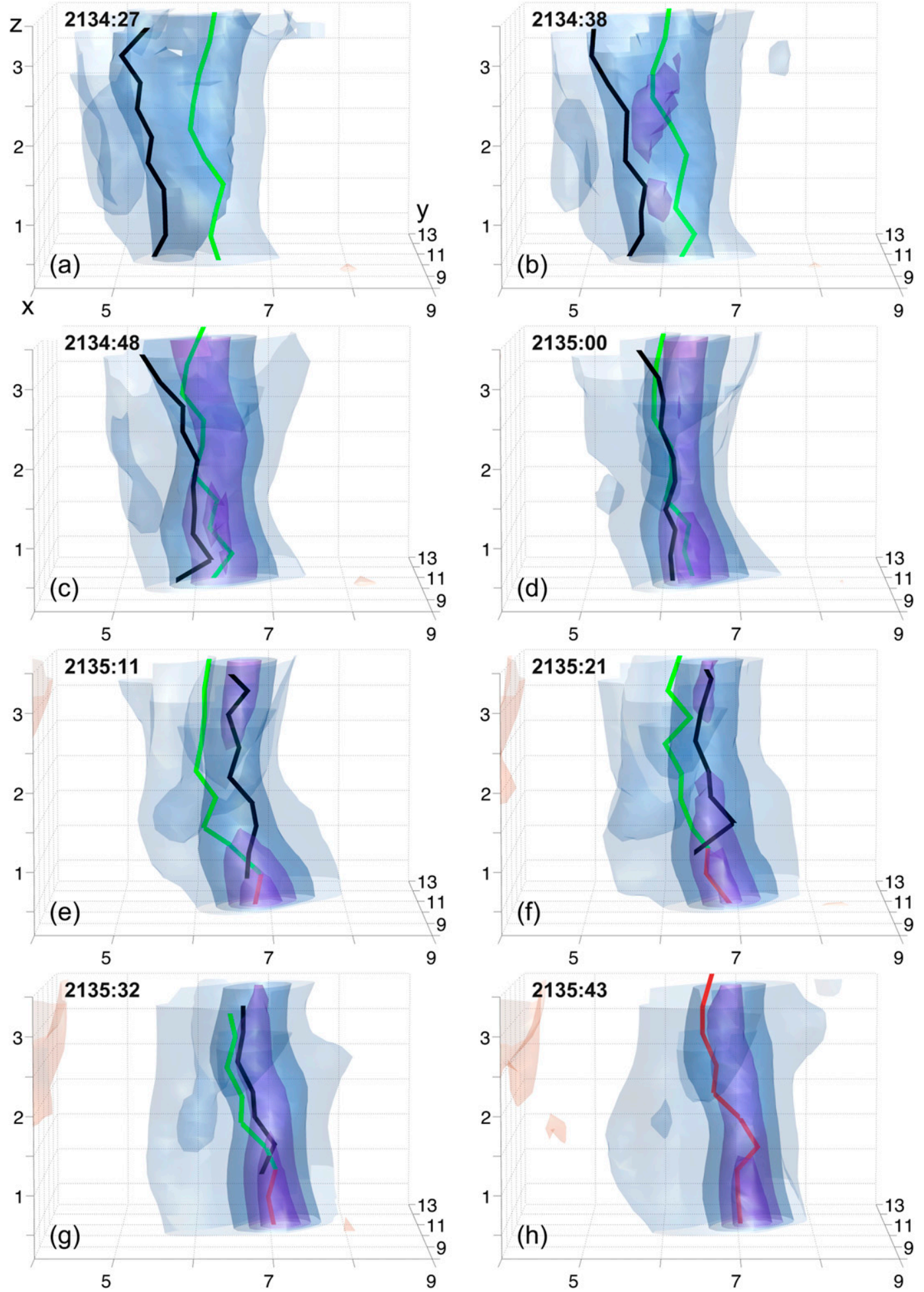

FIG. 11. Isosurfaces of azimuthal wind shear during the merger of the El Reno-Piedmont (green line), second (black line), and merged (red line) TVSs at (a) 2134:27, (b) 2134:38, (c) 2134:48, (d) 2135:00, (e) 2135:11, (f) 2135:21, (g) 2135:32, and (h) 2135:43 UTC 24 May 2011. The lines mark the estimated TVS center locations. Axes are labeled in (a). Cyclonic (anticyclonic) isosurfaces are plotted in purple (orange) every $0.015(-0.015) \mathrm{s}^{-1}$ beginning at 0.02 $(-0.02) \mathrm{s}^{-1}$. The view is from the south at an elevation angle of $6^{\circ}$. 

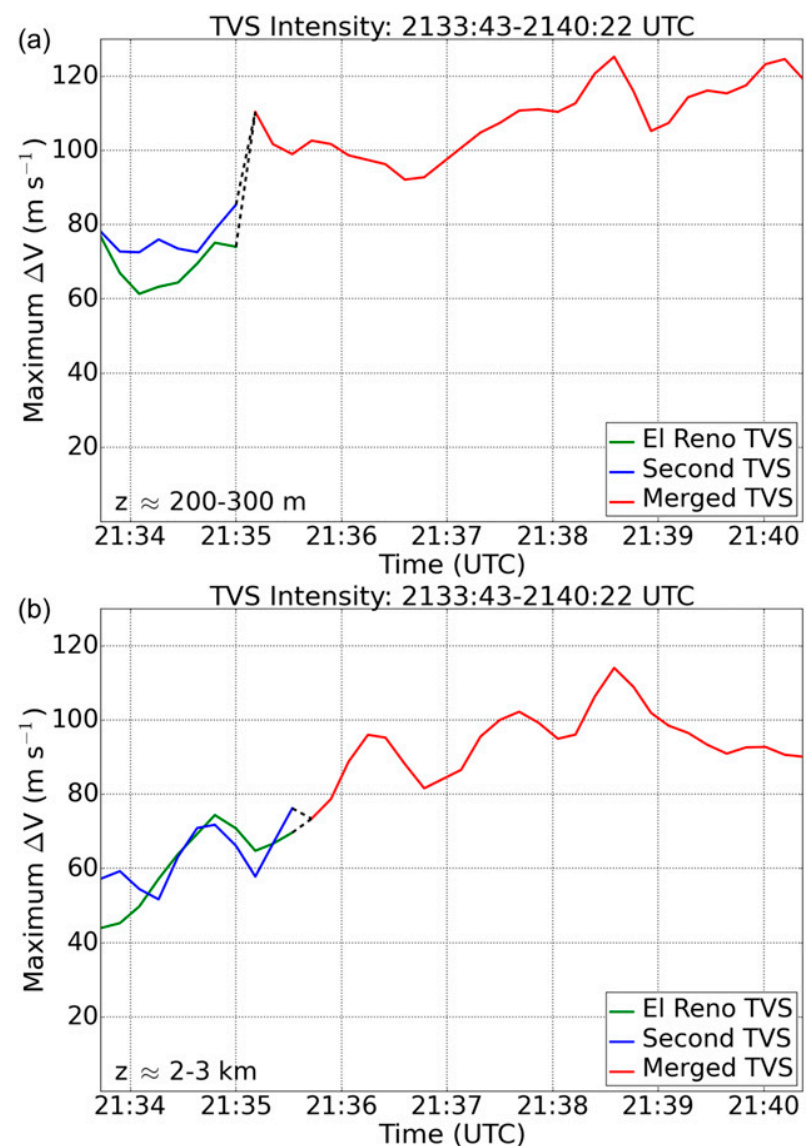

FIG. 12. Time series of maximum $\Delta V\left(\mathrm{~m} \mathrm{~s}^{-1}\right)$ in the El RenoPiedmont TVS, second TVS, and the subsequent merged TVS from MWR-05XP data at (a) $1.0^{\circ}$ and (b) $10.0^{\circ}$ elevation angle. The dotted black lines connect the last observations of simultaneous TVSs with the first observations of a merged TVS. The approximate heights of the observations are shown in the bottom left. A simple 1-2-1 filter was used to smooth the $\Delta V$ values.

\section{Summary and discussion}

The observations presented here offer a unique look at what could be a rare process. During the life cycle of the EF5 ERPT, a second tornado forms, strengthens, and then interacts and likely merges with the ERPT. Before T2 forms, the ERPT becomes increasingly displaced from the main updraft and TVS $\Delta V$ decreases a great amount. The $\mathrm{T} 2$ then forms north of both the ERPT and primary RFGF in an area where there is strong radial convergence, likely within the same midlevel mesocyclone as the ERPT. After T2 forms, the distance between the two TVSs decreases as they rotate around each other and then merge in a helical manner. Following the merger, there is an immediate increase in TVS intensity and size that lasts for several minutes. A definitive determination of why the storm evolves in this manner likely requires an inspection of high-resolution
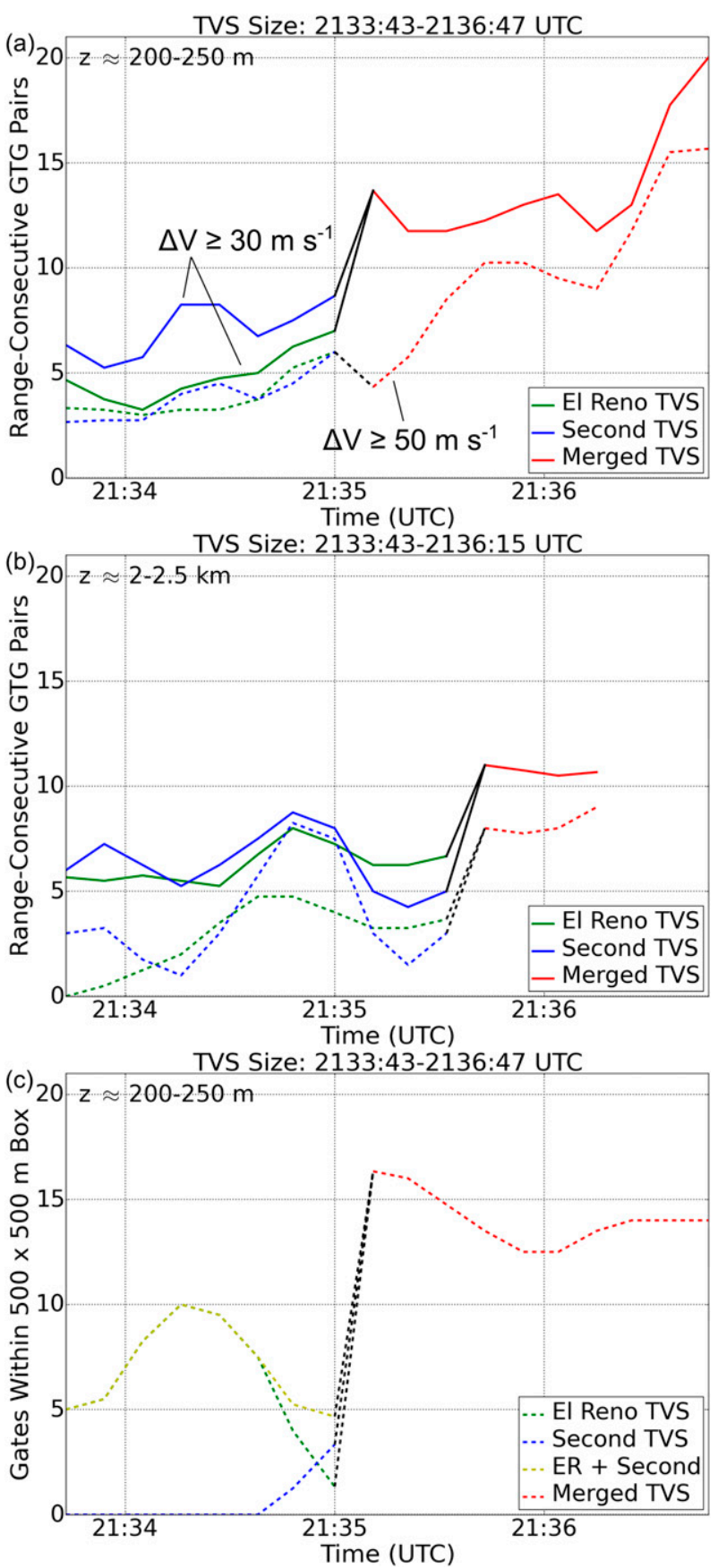

FIG. 13. Time series of the number of range-consecutive gates with $\Delta V \geq 30 \mathrm{~m} \mathrm{~s}^{-1}$ (solid lines) and $\Delta V \geq 50 \mathrm{~m} \mathrm{~s}^{-1}$ (dotted lines) in the El Reno-Piedmont, second, and subsequent merged TVS from MWR-05XP data at (a) $1.0^{\circ}$ and (b) $10.0^{\circ}$ elevation angle. (c) Number of gates in which radial velocity magnitude $\geq 50 \mathrm{~m} \mathrm{~s}^{-1}$ in two $500 \mathrm{~m} \times 500 \mathrm{~m}$ boxes centered on the maximum positive and negative radial velocity magnitude in each TVS. The black lines connect the last observations of simultaneous TVSs with the first observations of a merged TVS. The approximate heights of the observations are shown in the top left. A simple 1-2-1 filter was used to smooth the gate values. 
representations of the three-dimensional wind, pressure, and temperature fields, which are not available for this case. This supercell was simulated in Orf et al. (2014), but in an idealized framework, and the formation of $\mathrm{T} 2$ or a tornado merger was not captured. Possible mechanisms for the observed storm evolution are discussed below using the available data.

One might be tempted to categorize $\mathrm{T} 2$ as a satellite tornado. The tornado does meet several criteria of satellite tornadoes proposed recently in Edwards (2014): it forms within the same broad area of rotation as the ERPT (Fig. 7) and adjacent to a large violent tornado [though the ERPT was at a relative minimum in intensity immediately preceding the merger (French et al. 2014)]. In addition, the Edwards (2014) conceptual model proposes that satellite tornadoes usually form to the north of the main tornado as occurs in this case. However, the evolution in this case differs from that associated with most satellite tornadoes in that the two TVSs converge toward each other (Fig. 10) rather than having a satellite tornado converge toward a larger tornado. This behavior may be related to the similar size and intensity of the two TVSs (Figs. 12-13). In addition, despite possibly being within the same mesocyclone, T2 forms 5-7 km away from the ERPT (Fig. 3). One possibility is that $\mathrm{T} 2$ was an unusually large and intense satellite tornado that altered the evolution of the "typical" satellite tornado-main tornado interaction offered in Edwards (2014).

Likewise, the evolution of the TVSs in this case do not follow the cyclic tornadogenesis process as it has been observed previously, where the original tornado moves rearward and to the left of storm motion while the second tornado forms farther south, in a location where vertical vorticity generation and convergence are often maximized in a RFGF "bulge" (e.g., Burgess et al. 1982; Dowell and Bluestein 2002a). In this case, the ERPT moves at a similar speed and to the right of the storm in the 20 min preceding the merger (not shown), as has been documented in French et al. (2014) for two tornadoes prior to their dissipation. Also, T2 forms to the northwest rather than southeast of the ERPT (Fig. 3). However, this case does share several similarities with the conceptual model of "nonoccluding cyclic mesocyclogenesis" proposed in Adlerman and Droegemeier (2005, hereafter AD05). The T2 forms to the north of the ERPT near the intersection of the RFGF and forward-flank gust front boundaries (Figs. 3a,b) in the preferred location for a new low-level mesocyclone in the AD05 model. In addition, a sounding from Norman, Oklahoma, at 1800 sampled an environment about $40 \mathrm{~km}$ away within $2.5 \mathrm{~h}$ of the analysis time. There is a "quarter-circle" hodograph and $0-1 \mathrm{~km}\left(166 \mathrm{~m}^{2} \mathrm{~s}^{-2}\right)$ and $0-3 \mathrm{~km}\left(192 \mathrm{~m}^{2} \mathrm{~s}^{-2}\right)$ storm-relative helicity values consistent with the phase space occupied by nonoccluding cyclic mesocyclogenesis in the AD05 simulations (see their Fig. 21). The near-storm environment sampled by the sounding likely was different from that of the supercell. Nonetheless, another possibility is that nonoccluding cyclic tornadogenesis was occurring, but evolved differently such that, again, the tornadoes interacted rather than continue to move away from each other. Earlier in the life cycle of the same supercell, the dissipation of a brief tornado that preceded the ERPT also was consistent with the nonoccluding cyclic mesocyclogenesis process (Houser et al. 2015).

We believe the most intriguing aspect of this case is the similarities it shares with one of the only previously documented interaction cases: the Hesston and Goessel tornadoes in 1990 (Fujita 1992). In both cases, (i) the second tornado formed north of the original tornado, (ii) the first tornado shifted its track from northeastward to more northward prior to interacting and possibly merging with the second tornado, and (iii) the size (intensity) of the resulting tornado was larger (greater) than either of the original two tornadoes in the minutes prior to the interaction. These similarities provide for the possibility that the mechanism responsible for, and the effects of, the interaction and possible merger are similar for both cases. As such, we note here an additional case in which a possible merger on the tornado cyclone scale was observed (Lee et al. 2012). Similarly in that case, secondary tornado formation occurred to the north of the original tornado and the interaction and possible merger was followed by an increase in the size and intensity of the resulting tornado. A certain repeatable set of conditions in the environment and/or internal to the storm such as those that favor large satellite tornadoes or nonoccluding cyclic tornadogenesis could explain the similar evolution in these cases (if they exist). Additional possibilities for the interaction include that this process is an extreme example of a multiple vortex mesocyclone (Wurman and Kosiba 2013) or an incomplete or interrupted tornado dissipation that was coupled with a secondary genesis within a large mesocyclone.

In regards to the merger and subsequent increase in TVS intensity and size, the authors are not aware of any numerical modeling or theoretical work examining the merger of two tornadoes. However, several studies have simulated 2D vortex mergers to determine such effects generally. For example, it has been shown that two identical Rankine vortices that surpass a critical aspect ratio of core size to distance separation consistently merge (e.g., Melander et al. 1988; Waugh 1992; Brandt and Nomura 2006). If the vortices are unequal in size or 
intensity, as is often the case in nature, they frequently will not merge and/or undergo one of several more complicated interactions (e.g., Dritschel and Waugh 1992; Trieling et al. 2005). The merger of similarly sized vortices typically results in a vortex larger than either of the original vortices; in contrast, the merger of a larger vortex with a smaller vortex often will not lead to such an increase in size (Waugh 1992; Dritschel and Waugh 1992). If a complete vortex merger occurs, the core circulation of the merged vortex is likewise greater than that of either of the original two vortices.

In addition, in several studies, misocyclone mergers have been observed in severe storms (e.g., Roberts and Wilson 1995). In Lee and Wilhelmson (1997), simulated misocyclone mergers resulted in a misocyclone with greater circulation than either of the original misocyclones. In the high-resolution observations of Marquis et al. (2007), the merger of similarly sized misocyclones along boundaries were documented. The mergers typically resulted in a single, larger misocyclone, consistent with results from the 2D vortex simulations. Anecdotally, other mergers of vorticity maxima have been observed in supercells (e.g., Dowell and Bluestein 2002b) and in very high spatial resolution simulations of supercells (e.g., Schenkman et al. 2014; Orf et al. 2014). For example, in recent high spatial resolution simulations of a supercell (C. Potvin 2014, personal communication), a merger of two relativity strong small-scale vorticity maxima (each with $\zeta \geq 0.7 \mathrm{~s}^{-1}$ ) results in an increase in the circulation of the merged vortex by as much as $40 \%$ compared to the two individual vorticity maxima.

We speculate that the merger process and the effects of the merger on the resulting tornado in this case were supported by the similar size and intensity of the individual tornadoes at the time of the merger. The large distance $(>5 \mathrm{~km})$ between the two tornadoes when T2 formed may have allowed for $\mathrm{T} 2$ to develop in a favorable environment for strong tornadoes without interference from the ERPT. Because of the concurrent decrease in ERPT size and intensity during this time, there was an interaction between tornadoes with similar characteristics instead of having a dominant main tornado absorb a small, weak tornado with few lasting effects on size or intensity. We hesitate to further emphasize such comparisons because there likely are significant unresolved processes important in the merger process that only would be captured in large-eddy simulations of tornadoes (e.g., Lewellen et al. 2008).

Despite the presumed rarity of the events described in this case, we await further observations of tornado mergers to assess (i) the dynamical cause for such an evolution and (ii) the relative frequency with which such mergers intensify, enlarge, and extend the life cycles of tornadoes. The latter point, in particular, has potential implications for the eventual short-term forecasting and nowcasting of long-lived, violent tornadoes.

Acknowledgments. The authors thank Curtis Alexander, Corey Potvin, Alex Schenkman, and Jeffrey Snyder for useful data and discussions regarding this research topic. Robert Bluth at the Center for Interdisciplinary Remotely Piloted Aircraft Studies (CIRPAS) supported field operations with the MWR-05XP. We are grateful to Paul Buczynski and Randy George for their assistance in MWR-05XP data collection. We acknowledge the ongoing efforts of NSSL/CIMMS staff, including David Priegnitz and Pam Heinselman, in obtaining KOUN and MPAR data. James Hilger graciously provided video of the El Reno-Piedmont tornado. Unidata's Integrated Data Viewer was used to compare radar data to the surveyed damage path. Comments from Jim Marquis, Roger Edwards, and one anonymous reviewer improved several key points. Roger Edwards also provided photographs of the event. This work was completed while the first author was a National Research Council Postdoctoral Research Fellow at the National Severe Storms Laboratory.

\section{REFERENCES}

Adlerman, E. J., and K. K. Droegemeier, 2005: The dependence of numerically simulated cyclic mesocyclogenesis upon environmental vertical wind shear. Mon. Wea. Rev., 133, 35953623, doi:10.1175/MWR3039.1.

Alexander, C. R., and J. Wurman, 2005: The 30 May 1998 Spencer, South Dakota, storm. Part I: The structural evolution and environment of the tornadoes. Mon. Wea. Rev., 133, 72-97, doi:10.1175/MWR-2855.1.

Barnes, S. L., 1964: A technique for maximizing details in numerical weather map analysis. J. Appl. Meteor., 3, 396-409, doi:10.1175/1520-0450(1964)003<0396:ATFMDI > 2.0.CO;2.

Bluestein, H. B., M. M. French, R. L. Tanamachi, S. Frasier, K. Hardwick, F. Junyent, and A. L. Pazmany, 2007: Closerange observations of tornadoes in supercells made with a dual-polarization, X-band, mobile Doppler radar. Mon. Wea. Rev., 135, 1522-1543, doi:10.1175/MWR3349.1.

- — - I. PopStefanija, R. T. Bluth, and J. B. Knorr, 2010: A mobile, phased-array Doppler radar for the study of severe convective storms: The MWR-05XP. Bull. Amer. Meteor. Soc., 91, 579-600, doi:10.1175/2009BAMS2914.1.

, J. C. Snyder, K. J. Thiem, Z. B. Wienhoff, J. B. Houser, and V. Mahale, 2014: Recent observations of tornadoes using a mobile, rapid-scan, polarimetric, X-band, Doppler radar. Eighth European Conf. on Radar in Meteorology and Hydrology, Garmisch-Partenkirchen, Germany, Institut für Physik der Atmosphäre, 10.6. [Available online at http://www.pa.op.dlr.de/ erad2014/programme/ExtendedAbstracts/012_Bluestein.pdf.]

Bodine, D. J., M. R. Kumjian, R. D. Palmer, P. L. Heinselman, and A. V. Ryzhkov, 2013: Tornado damage estimation using polarimetric radar. Wea. Forecasting, 28, 139-158, doi:10.1175/ WAF-D-11-00158.1. 
Brandt, L. K., and K. K. Nomura, 2006: The physics of vortex merger: Further insight. Phys. Fluids, 18, 051701, doi:10.1063/ 1.2201474

Brown, R. A., L. R. Lemon, and D. W. Burgess, 1978: Tornado detection by pulsed Doppler radar. Mon. Wea. Rev., 106, 29-39, doi:10.1175/1520-0493(1978)106<0029:TDBPDR>2.0.CO;2.

Burgess, D. W., V. T. Wood, and R. A. Brown, 1982: Mesocyclone evolution statistics. Preprints, 12th Conf. on Severe Local Storms, San Antonio, TX, Amer. Meteor. Soc., 422-424.

Conway, J. W., and D. S. Zrnić, 1993: A study of embryo production and hail growth using dual-Doppler and multiparameter radar. Mon. Wea. Rev., 121, 2511-2528, doi:10.1175/ 1520-0493(1993)121<2511:ASOEPA > 2.0.CO;2.

Davies, J. M., C. A. Doswell, D. W. Burgess, and J. F. Weaver, 1994: Some noteworthy aspects of the Hesston, Kansas, tornado family of 13 March 1990. Bull. Amer. Meteor. Soc., 75, 1007-1017, doi:10.1175/1520-0477(1994)075<1007: SNAOTH $>2.0 . \mathrm{CO} ; 2$.

Dowell, D. C., and H. B. Bluestein, 2002a: The 8 June 1995 McLean, Texas, storm. Part I: Observations of cyclic tornadogenesis. Mon. Wea. Rev., 130, 2626-2648, doi:10.1175/ 1520-0493(2002)130<2626:TJMTSP > 2.0.CO;2.

_ , and —_, 2002b: The 8 June 1995 McLean, Texas, storm. Part II: Cyclic tornado formation, maintenance, and dissipation. Mon. Wea. Rev., 130, 2649-2670, doi:10.1175/ 1520-0493(2002)130<2649:TJMTSP > 2.0.CO;2.

Dritschel, D. G., and D. W. Waugh, 1992: Quantification of the inelastic interaction of unequal vortices in two-dimensional vortex dynamics. Phys. Fluids, 4, 1737-1744, doi:10.1063/ 1.858394.

Edwards, R., 2014: Characteristics of supercellular satellite tornadoes. 27th Conf. on Severe Local Storms, Madison, WI, Amer. Meteor. Soc., 17.5. [Available online at https://ams.confex. com/ams/27SLS/webprogram/Paper254326.html.]

French, M. M., H. B. Bluestein, I. PopStefanija, C. Baldi, and R.T. Bluth, 2013: Reexamining the vertical development of tornadic vortex signature in supercells. Mon. Wea. Rev., 141, 45764601, doi:10.1175/MWR-D-12-00315.1.

,,,$--- \ldots$, and,- 2014 : Mobile, phased-array, Doppler radar observations of tornadoes at $\mathrm{X}$ band. Mon. Wea. Rev., 142, 1010-1036, doi:10.1175/MWR-D-13-00101.1.

Fujita, T. T., 1992: Memoirs of an Effort to Unlock the Mystery of Severe Storms during the 50 Years, 1942-1992. University of Chicago, 298 pp.

— D. L. Bradbury, and C. F. Van Thullenar, 1970: Palm Sunday tornadoes of April 11, 1965. Mon. Wea. Rev., 98, 29-69, doi:10.1175/1520-0493(1970)098<0029:PSTOA > 2.3.CO;2.

Fujiwhara, S., 1931: Short note on the behavior of two vortices. Proc. Phys. Math. Soc. Japan, 13, 106-110.

Grazulis, T. P., 1997: Significant Tornadoes, Update: 1992-1995. Environmental Films, 117 pp.

Houser, J. L., H. B. Bluestein, and J. C. Snyder, 2015: Rapid-scan, polarimetric, Doppler radar observations of tornadogenesis and tornado dissipation in a tornadic supercell: The "El Reno, Oklahoma" storm of 24 May 2011. Mon. Wea. Rev., 143, 27092734, doi:10.1175/MWR-D-14-00253.

Illingworth, A. J., J. W. F. Goddard, and S. M. Cherry, 1987: Polarization radar studies of precipitation development in convective storms. Quart. J. Roy. Meteor. Soc., 113, 469-489, doi:10.1002/ qj. 49711347604.

Kumjian, M. R., and A. V. Ryzhkov, 2008: Polarimetric signatures in supercell thunderstorms. J. Appl. Meteor. Climatol., 47, 1940-1961, doi:10.1175/2007JAMC1874.1.
- A. P. Khain, N. Benmoshe, E. Ilotoviz, A. V. Ryzhkov, and V. T. J. Phillips, 2014: The anatomy and physics of $Z_{D R}$ columns: Investigating a polarimetric radar signature with a spectral bin microphysical model. J. Appl. Meteor. Climatol., 53, 1820-1843, doi:10.1175/JAMC-D-13-0354.1.

Lee, B. D., and R. B. Wilhelmson, 1997: The numerical simulation of non-supercell tornadogenesis. Part I: Initiation and evolution of pretornadic misocyclone circulations along a dry outflow boundary. J. Atmos. Sci., 54, 32-60, doi:10.1175/ 1520-0469(1997)054<0032:TNSONS >2.0.CO;2.

_ C. A. Finley, and C. D. Karstens, 2012: The Bowdle, South Dakota, cyclic tornadic supercell of 22 May 2010: Surface analysis of rear-flank downdraft evolution and multiple internal surges. Mon. Wea. Rev., 140, 3419-3441, doi:10.1175/ MWR-D-11-00351.1.

Lewellen, D. C., B. Gong, and W. S. Lewellen, 2008: Effects of finescale debris on near-surface tornado dynamics. J. Atmos. Sci., 65, 3247-3262, doi:10.1175/2008JAS2686.1.

Majcen, M., P. Markowski, Y. Richardson, D. Dowell, and J. Wurman, 2008: Multipass objective analysis of Doppler radar data. J. Atmos. Oceanic Technol., 25, 1845-1858, doi:10.1175/2008JTECHA1089.1.

Marquis, J. N., Y. P. Richardson, and J. M. Wurman, 2007: Kinematic observations of misocyclones along boundaries during IHOP. Mon. Wea. Rev., 135, 1749-1768, doi:10.1175/MWR3367.1.

Melander, M. V., N. J. Zabusky, and J. C. Mcwilliams, 1988: Symmetric vortex merger in two dimensions: Causes and conditions. J. Fluid Mech., 195, 303-340, doi:10.1017/ S0022112088002435.

Orf, L., R. B. Wilhelmson, L. J. Wicker, B. D. Lee, and C. A. Finley, 2014: Genesis and maintenance of a long-track EF5 tornado embedded within a simulated supercell. 27th Conf. on Severe Local Storms, Madison, WI, Amer. Meteor. Soc., 3B.3. [Available online at https://ams.confex.com/ams/27SLS/ webprogram/Paper255451.html.]

Pazmany, A. L., J. B. Mead, H. B. Bluestein, J. C. Snyder, and J. B. Houser, 2013: A mobile, rapid-scanning, X-band, polarimetric (RaXPol) Doppler radar system. J. Atmos. Oceanic Technol., 30, 1398-1413, doi:10.1175/JTECH-D-12-00166.1.

Rasmussen, E. N., J. M. Straka, R. Davies-Jones, C. A. Doswell III, F. H. Carr, M. D. Eilts, and D. R. MacGorman, 1994: Verification of the Origins of Rotation in Tornadoes Experiment: VORTEX. Bull. Amer. Meteor. Soc., 75, 995-1006, doi:10.1175/ 1520-0477(1994)075<0995:VOTOOR >2.0.CO;2.

Roberts, R. D., and J. W. Wilson, 1995: The genesis of three nonsupercell tornadoes observed with dual-Doppler radar. Mon. Wea. Rev., 123, 3408-3436, doi:10.1175/1520-0493(1995)123<3408: TGOTNT $>2.0 . \mathrm{CO} ; 2$.

Ryzhkov, A., D. Burgess, D. Zrnić, T. Smith, and S. Giangrande, 2002: Polarimetric analysis of a 3 May 1999 tornado. Preprints, 21th Conf. on Severe Local Storms, San Antonio, TX, Amer. Meteor. Soc., 14.2. [Available online at https://ams.confex. com/ams/SLS_WAF_NWP/techprogram/paper_47348.htm.]

—, T. J. Schuur, D. W. Burgess, and D. S. Zrnić, 2005: Polarimetric tornado detection. J. Appl. Meteor., 44, 557-570, doi:10.1175/JAM2235.1.

Schenkman, A. D., M. Xue, and M. Hu, 2014: Tornadogenesis in a high-resolution simulation of the 8 May 2003 Oklahoma City supercell. J. Atmos. Sci., 71, 130-154, doi:10.1175/ JAS-D-13-073.1.

Snyder, J. C., H. B. Bluestein, V. Venkatesh, and S. J. Frasier, 2013: Observations of polarimetric signatures in supercells by an X-band 
mobile Doppler radar. Mon. Wea. Rev., 141, 3-29, doi:10.1175/ MWR-D-12-00068.1.

Speheger, D. A., C. A. Doswell, and G. J. Stumpf, 2002: The tornadoes of 3 May 1999: Event verification in central Oklahoma and related issues. Wea. Forecasting, 17, 362-381, doi:10.1175/ 1520-0434(2002)017<0362:TTOMEV>2.0.CO;2.

Tanamachi, R. L., H. B. Bluestein, J. B. Houser, S. J. Frasier, and K. M. Hardwick, 2012: Mobile, X-band, polarimetric Doppler radar observations of the 4 May 2007 Greensburg, Kansas, tornadic supercell. Mon. Wea. Rev., 140, 2103-2125, doi:10.1175/ MWR-D-11-00142.1.

Trieling, R. R., O. U. Velasco Fuentes, and G. J. F. van Heijst, 2005 Interaction of two unequal corotating vortices. Phys. Fluids, 17, 087103, doi:10.1063/1.1993887.

Ward, N. B., 1972: The exploration of certain features of tornado dynamics using a laboratory model. J. Atmos. Sci., 29, 1194-1204, doi:10.1175/1520-0469(1972)029<1194:TEOCFO>2.0.CO;2.
Waugh, D. W., 1992: The efficiency of symmetric vortex merger. Phys. Fluids, 4, 1745-1758, doi:10.1063/1.858395.

Wood, V. T., and R. A. Brown, 1997: Effects of radar sampling on single-Doppler velocity signatures of mesocyclones and tornadoes. Wea. Forecasting, 12, 928-938, doi:10.1175/ 1520-0434(1997)012<0928:EORSOS>2.0.CO;2.

Wurman, J., and S. Gill, 2000: Finescale radar observations of the Dimmitt, Texas (2 June 1995), tornado. Mon. Wea. Rev., 128, 2135-2164, doi:10.1175/1520-0493(2000)128<2135: FROOTD $>2.0 . \mathrm{CO} ; 2$.

— , and K. Kosiba, 2013: Finescale radar observations of tornado and mesocyclone structures. Wea. Forecasting, 28, 1157-1174, doi:10.1175/WAF-D-12-00127.1.

Zrnić, D. S., and Coauthors, 2007: Agile-beam phased array radar for weather observations. Bull. Amer. Meteor. Soc., 88, 17531766, doi:10.1175/BAMS-88-11-1753. 